\title{
Understanding the Impact of Return-Current Losses on the X-Ray Emission from Solar Flares
}

\author{
Gordon D. Holman \\ NASA Goddard Space Flight Center, Code 671, Greenbelt, MD 20771 \\ Gordon.D.Holman@nasa.gov
}

Received —

Submitted to The Astrophysical Journal 


\begin{abstract}
I obtain and examine the implications of one-dimensional analytic solutions for return-current losses on an initially power-law distribution of energetic electrons with a sharp low-energy cutoff in flare plasma with classical (collisional) resistivity. These solutions show, for example, that return-current losses are not sensitive to plasma density, but are sensitive to plasma temperature and the lowenergy cutoff of the injected nonthermal electron distribution. A characteristic distance from the electron injection site, $x_{r c}$, is derived. At distances less than $x_{r c}$ the electron flux density is not reduced by return-current losses, but plasma heating can be substantial in this region, in the upper, coronal part of the flare loop. Before the electrons reach the collisional thick-target region of the flare loop, an injected power-law electron distribution with a low-energy cutoff maintains that structure, but with a flat energy distribution below the cutoff energy, which is now determined by the total potential drop experienced by the electrons. Modifications due to the presence of collisional losses are discussed. I compare these results with earlier analytical results and with more recent numerical simulations. Emslie's 1980 conjecture that there is a maximum integrated X-ray source brightness on the order of $10^{-15}$ photons $\mathrm{cm}^{-2} \mathrm{~s}^{-1} \mathrm{~cm}^{-2}$ is examined. I find that this is not actually a maximum brightness and its value is parameter dependent, but it is nevertheless a valuable benchmark for identifying return-current losses in hard X-ray spectra. I discuss an observational approach to identifying return-current losses in flare data, including identification of a return-current "bump" in X-ray light curves at low photon energies.
\end{abstract}

Subject headings: acceleration of particles — plasmas — Sun: flares — Sun: X-rays, gamma rays 


\section{Introduction}

Solar flares, the most energetic explosive events in the solar system, provide a nearby laboratory for developing our understanding of the physics of evolving, magnetized cosmic plasma. Because of their proximity to Earth, they and related coronal mass ejections (CMEs) have an increasingly significant impact on the power grid, spacebased communications and other equipment, and manned space programs. Therefore, understanding the phenomenology and physics of solar flares is of considerable academic and practical interest.

Accelerated electrons play an important role in the energetics of solar flares. Understanding the process or processes that accelerate these electrons to high, suprathermal energies depends in part on understanding the evolution of the electrons between the acceleration region and the regions where they are observed through their hard X-ray or radio emission (see Holman et al. 2011, for a review). Hard X-ray emission is usually observed from the chromosphere and lower transition region, where the plasma density in the solar atmosphere is highest. This is where they interact most frequent with ambient ions, giving the highest flux of bremsstrahlung X-rays, and where they lose through collisions their suprathermal energy and collisionally heat the ambient plasma. Thus the region from which the X-ray emission is greatest is a thick target. The primary particle acceleration region, however, is understood to be in the corona, above the hot soft X-ray and EUV loops observed in flares. Radio emission is observed from the corona, but its location and properties strongly depend on the magnetic field strength and structure. In some flares hard X-ray emission is also observed from the corona, possibly even from the acceleration region itself, but the proper interpretation of this coronal emission remains elusive.

Theoretical arguments and observational results indicate that return-current energy 
losses can have a significant impact on electrons accelerated in flares. Recognizing that the high current in the beam of energetic electrons required to explain observed X-ray fluxes would be pinched off by its associated magnetic field, Hoyng et al. (1976) argued that the current must be neutralized by a return-current in the background plasma. Flare particle acceleration mechanisms are not expected to produce co-streaming ions to neutralize the beam of accelerated electrons (e.g., Miller et al. 1997). Also, Ramaty High Energy Spectrosocpic Imager (RHESSI) observations have indicated that the $\gamma$-ray emission from ions originates from different locations than that from electrons (Hurford et al. 2003, 2006). For the electron beam to successfully propagate away from the acceleration region and for charge conservation to be maintained, a co-spatial return current is required.

The return current is driven by an electric field induced in the plasma by the streaming, energetic electrons in the beam (van den Oord 1990, and references therein). This electric field, in addition to driving the return current, also extracts energy from the beam electrons. The return current collisionally heats the plasma through Joule heating. As the beam electrons lose energy, the current they carry, the return current, and the return-current electric field decrease with propagation distance. To understand the impact of return-current losses on flare hard X-ray emission, it is important to determine how these losses change the electron distribution function with distance from the acceleration region.

A steady-state, one-dimensional (1-D, all electron velocities have zero pitch angle and, therefore, are directed along the magnetic field) model for the beam/return-current system was developed by Knight \& Sturrock (1977). They apply their model to a discussion of the additional heating of the flare plasma by the return current. Emslie (1980) extended the model to include a non-zero electron pitch angle and collisional losses. He computed the evolution of electron energy and pitch angle with depth (column density) and deduced the existence of an upper limit to the flare X-ray emission. D'Iakonov \& Somov (1988) 
estimated the hard X-ray spectrum and polarization of the bremsstrahlung radiation from a hot, thermal tail of electrons escaping ahead of a thermal conduction front. Return-current losses were included in their calculations. Litvinenko \& Somov (1991) repeated these calculations for a power-law distribution of electrons. The impact of the shape of the low-energy cutoff to the energy distribution of the injected electrons on both collisional and return-current heating of the flare plasma has been studied by Brown \& McClymont (1987).

Numerical simulations of electron propagation with return-current losses and computations of corresponding hard X-ray spectra have been performed by Zharkova \& Gordovskyy (2005, 2006). For an injected power-law electron energy distribution with lowand high-energy cutoffs, they computed the steady-state electron distribution along the flare loop and the resulting bremsstrahlung X-ray spectrum for several injected power-law indices and electron energy flux densities. These computations included the response of the flare plasma to the electron beam injection. Subsequent simulations (Siversky \& Zharkova 2009; Zharkova et al. 2010) have included time-dependence of the particle injection (see also Karlicky et al. 1990), electron pitch-angle anisotropy, and magnetic field convergence. Papers addressing plasma instabilities in the electron beam/return-current system include Emslie (1981), Rowland \& Vlahos (1985), Karlický et al. (2008), Lee et al. (2008), Karlický (2009), Karlický \& Kašparová (2009), and Zharkova \& Siversky (2011).

Observational evidence exists indicating that in some cases return-current losses do have a significant impact on the hard X-ray emission from flares: (1) Sui et al. (2007) found that the spectral break energy (and, therefore, the corresponding electron distribution cutoff energy), was correlated with the X-ray flux for a sample of flares having relatively flat, low-energy spectra. This correlation is expected if return-current losses are responsible for the spectral break (see Sections 2.2 and 3.3 of this paper). (2) Alexander \& Daou (2007) have found that the integrated hard X-ray flux density (above $20 \mathrm{keV}$ ) does not 
monotonically increase with flare size, but levels off to a maximum value as the flare size becomes large. This maximum value agrees with the upper limit deduced by Emslie (1980). (3) Battaglia \& Benz (2008) found that for two flares with well-observed coronal X-ray emission, the difference between the spectral indices of the coronal and footpoint spectra was greater than the largest difference expected in a simple thick-target model. They argued that return-current losses were most likely responsible for this large difference between the spectral indices.

Developing the ability to recognize the impact of return-current losses on flare hard X-ray data is a crucial step to understanding electron acceleration and propagation in flares. The energy spectrum of electrons injected from the acceleration region cannot be confidently established without first understanding when and how return-current losses affect the observed X-ray spectrum. Identifying return-current losses in turn provides information about the induced potential drop in flare loops and its evolution and, consequently, about the evolution of the plasma in the loops. This plasma evolution is coupled to the direct collisional and indirect return-current heating of the plasma by the accelerated electrons. Hence, return-current losses contribute to the flare plasma dynamics.

Although considerable work has been done on the origin of the return current and its consequences, it is difficult to obtain from the existing literature a clear understanding of how to recognize and interpret return-current losses in flare X-ray data. The purpose of this paper is to derive relatively simple analytical and semi-analytical results for return-current losses, with physical understanding and application to flare observations as primary goals, although no direct application to flare X-ray data is included in this paper. Numerical results are obtained throughout for estimating the impact of the losses. The analytical results obtained here are also useful as limiting cases for testing numerical codes.

This paper focuses on a one-dimensional (1-D), steady-state model, where 1-D 
means that all electron velocities are directed along the loop magnetic field. These same assumptions are usually included in the thick-target model (Brown 1971). The model is the same as that of Knight \& Sturrock (1977), but with a minor but generally important extension to approximate the thermalization of electrons as their energies approach the mean energy of electrons in the ambient thermal plasma. Except for this thermalization, collisional losses are not incorporated into the model. This model is adequate for understanding the primary qualitative effects of return-current losses and, as I show in Section 3, for understanding the qualitative (and, to some extent, quantitative) results of Zharkova \& Gordovskyy (2006). Since the model is 1-D, it does not include the evolution of electron pitch angles and the re-acceleration of reflected electrons (e.g., Karlicky 1993). It can accommodate a non-classical value of the plasma resistivity, but not significant physical changes in the particle distributions and their evolution due to plasma kinetic effects, such as acceleration of electrons out of the thermal plasma as the return-current electric field approaches and exceeds the Dreicer field (e.g., Karlický et al. 2004).

In Section 2 the model is described and the electron flux density distribution function and all related physical quantities are derived. Formalism for generalizing these results to the more realistic case of plasma resistivity varying with distance is derived. Heating of the plasma by the beam/return-current system (excluding collisional heating by the higher-energy primary beam electrons) is derived and discussed. Return-current and collisional energy losses are compared. The impact of return-current losses on X-ray source brightness is discussed, and I introduce a model in which collisional losses dominate in the thick-target region and return-current losses dominate above this region. An approximation to the X-ray brightness spectrum from this model is computed. I also examine the maximum integrated X-ray brightness deduced by Emslie (1980) and find that this is not a maximum value, but rather is characteristic of the integrated brightness when return-current losses begin to degrade the flux density of the electron beam. Finally, a much higher limit on the 
maximum integrated X-ray brightness is identified and discussed.

An analysis and interpretation of the numerical results obtained by Zharkova \& Gordovskyy (2006) is presented in Section 3. I analyze and interpret results for the return-current electric field, X-ray spectra, and X-ray spectral indices.

The evolution of the return-current low-energy cutoff (potential drop) and X-ray spectral index with X-ray brightness, and of brightness with injected electron flux density, are derived from the model in Section 4. The results are examined and a new feature, the return-current "bump", is identified.

The model results are summarized in Section 5 and, based on the results obtained here, an approach to identifying and interpreting return-current losses in flare X-ray data to deduce physical information is discussed.

\section{Steady-State 1-D Model}

The steady-state, one-dimensional model is based on the following assumptions:

1. All electron velocities are parallel (or all are anti-parallel) to the ambient magnetic field, as in the collisional thick-target model of Brown (1971). The velocities of the accelerated electrons are parallel to the direction of the return-current electric field.

2. The magnitude of the return-current density, $J_{r c}$ statampere $\mathrm{cm}^{-2}$ in cgs units, has had time to reach its steady-state value, and this is equal to the magnitude of the current density $J$ of the accelerated electrons. The time required to reach the steady state is on the order of the thermal electron-ion collision time (van den Oord 1990) which, from Huba (2009), is

$$
\tau_{e}=\frac{3}{4}\left(\frac{m_{e}}{2 \pi}\right)^{1 / 2} \frac{(k T)^{3 / 2}}{n \lambda e^{4}}=\frac{4.35 \times 10^{-2} T_{7}^{3 / 2}}{n_{10}(\lambda / 20)} \mathrm{s} .
$$


Here $m_{e}$ and $e$ are the electron mass and charge, respectively, and $k$ is Boltzmann's constant. $T$ and $n$ are the plasma electron temperature $(\mathrm{K})$ and density $\left(\mathrm{cm}^{-3}\right)$, respectively, and $\lambda$ is the Coulomb logarithm. Notation such as $T_{7}$ is shorthand for $\left(\mathrm{T} / 10^{7}\right)$, meaning that the temperature is normalized to the value $10^{7} \mathrm{~K}$. The Coulomb logarithm is approximately (with $\sim 10 \%$ uncertainty)

$$
\begin{aligned}
\lambda=8.96-\ln \left(Z n^{1 / 2} T^{-3 / 2}\right), & T<1.16 \times 10^{5} Z^{2} \\
\lambda=14.6+\ln \left(n^{-1 / 2} T\right), & T>1.16 \times 10^{5} Z^{2} .
\end{aligned}
$$

The atomic number $Z$ in the solar atmosphere is approximately the relativeion-abundance-weighted mean 1.1, and the weighted mean $Z^{2}$ is approximately 1.4 .

In the flare corona, taking $T$ to range from $1 \mathrm{MK}$ to $50 \mathrm{MK}$ and and $n$ from $1 \times 10^{9} \mathrm{~cm}^{-3}$ to $1 \times 10^{12} \mathrm{~cm}^{-3}, \lambda$ ranges from 15 to 22 and $\tau_{e}$ ranges from $19 \mu \mathrm{s}$ to $4.4 \mathrm{~s}$. Below the corona, with $T$ ranging from $10^{4} \mathrm{~K}$ to $1 \mathrm{MK}$ and and $n$ from $1 \times 10^{9} \mathrm{~cm}^{-3}$ to $1 \times 10^{14} \mathrm{~cm}^{-3}, \lambda$ ranges from 7 to 18 and $\tau_{e}$ ranges from $42 \mathrm{~ns}$ to $15 \mathrm{~ms}$. The steady-state assumption will be violated if the accelerated electron distribution varies on timescales on the order of $\tau_{e}$ or less.

3. The resistivity of the plasma, $\eta$, is given by the classical, collisional Spitzer result

$$
\eta=\frac{m_{e}}{1.96 n e^{2} \tau_{e}}=0.680\left(2 \pi m_{e} e^{4}\right)^{1 / 2} \lambda(k T)^{-3 / 2}=4.63 \times 10^{-18}\left(\frac{\lambda}{20}\right) T_{7}^{-3 / 2} \quad \mathrm{~s}
$$

where $m_{e}$ and $e$ are the electron mass and charge, respectively (Huba 2009). Plasma instabilities may result in a plasma resistivity exceeding this value and/or more complex behavior in the beam-return-current system, especially when the drift speed of the return current exceeds the ion sound speed. Since the purpose of this paper is to better understand the impact of classical return-current losses on flare observational data and to compare derived results with numerical simulations of return-current 
losses under classical conditions, I will assume that the plasma resistivity is given by Eq. 3, even when instability is likely to affect the beam-return-current system.

With these assumptions, the strength of the electric field driving the return current, $E_{r c}$ statvolt $\mathrm{cm}^{-1}$, is given by Ohm's law:

$$
E_{r c}=\eta J_{r c}=\eta J
$$

4. The electrons are continuously injected from the acceleration region, taken to be located at position $x=0$, and have a power-law flux density distribution in electron energy, $E \mathrm{keV}$, with a low-energy cutoff, $E_{c} \mathrm{keV}$ :

$$
F(E, x=0)= \begin{cases}(\delta-1) E_{c}^{\delta-1} F_{e 0} E^{-\delta} \text { electrons } \mathrm{s}^{-1} \mathrm{~cm}^{-2} \mathrm{keV}^{-1} & E \geq E_{c} \\ 0 & E<E_{c} .\end{cases}
$$

Here $\delta$ is the power-law index of the electron distribution and $F_{e 0}$ (electrons s ${ }^{-1}$ $\left.\mathrm{cm}^{-2}\right)=\int_{0}^{\infty} F(E, 0) d E$ is the total flux density of accelerated electrons. The power-law index $\delta$ is greater than 2 so that the total energy flux density carried by the accelerated electrons is not infinite (see Holman et al. 2011). In this section I assume that any high-energy cutoff to this distribution function is high enough that it does not significantly affect the value of $F_{e 0}$ and related quantities. A high-energy cutoff is considered in Sections 3.2 and 3.3.

5. Only return-current losses are considered. In particular, collisional losses are not included. This is done to isolate effects specifically due to return-current losses and to allow relatively simple, analytical results to be obtained. The impact of collisional losses on return-current losses is discussed in Section 2.5. Modifications to the electron distribution function by other likely processes, such as electron pitch-angle scattering and magnetic mirroring, are also not included.

6. When an accelerated electron's energy is reduced by the return-current losses to a value $E_{t h}$, it is assumed to be lost from the electron distribution. When the electrons 
lose sufficient energy, they will be thermalized into the ambient plasma. This will occur at energies somewhat greater than $k T$, where $k$ is Boltzmann's constant and $T$ is the temperature of the ambient plasma. I take this to be the discrete energy $E_{t h}=\beta k T$, where $\beta$ is a constant greater than but on the order of 1 . Emslie (2003) found that thermalization starts becoming significant when $E<5 k T$, giving

$1<\beta<5$. The effective value of $\beta$ is most likely $\sim 2-3$. I will assume that above $E_{t h}$ the electron flux density is conserved (see Section 2.2).

\subsection{Return-Current Electric Field Electron Deceleration}

The energy lost by the accelerated electrons in the return-current electric field $E_{r c}$ as a function of distance $x$ from the point of injection at $x=0$ is determined by the simple equation

$$
\frac{d E}{d x}=-e E_{r c}(x)
$$

The solution to this equation is

$$
E=E_{0}-e \int_{0}^{x} E_{r c}(x) d x=E_{0}-V(x) .
$$

Here $E_{0}$ is the initial value of the electron energy at $x=0$ and $V(x)$ is the potential drop (in $\mathrm{kV}$ when the electron energies are given in $\mathrm{keV}$ ) from $x=0$ to $x$. Note that the energy lost at position $x$ is the same for all electrons and equal to the value of the potential drop. However, the fractional energy lost, $V(x) / E_{0}$, is greater for lower energy electrons than for higher energy electrons. $V(x)$ is an increasing function of $x$.

Since the direction of current is defined by the collective motion of positive charge carriers, the current carried by the accelerated electrons is in the $-x$ direction while the return current and the return-current electric field are in the $+x$ direction. The current density carried by the accelerated electrons is $J(x)=-e \int_{0}^{\infty} F(E, x) d E$. I will usually drop 
the sign and refer only to the magnitude of the current, as in Equation 4.

Ohm's law (Equation 4) and Equation 3 can now be used to compute the magnitude of $E_{r c}$ at $x=0$ :

$$
\begin{gathered}
E_{r c 0} \equiv E_{r c}(0)=\eta J(0)=\eta e F_{e 0}=2.22 \times 10^{-27}\left(\frac{\lambda}{20}\right) T_{7}^{-3 / 2} F_{e 0} \text { statvolt } \mathrm{cm}^{-1} \\
=6.67 \times 10^{-28}\left(\frac{\lambda}{20}\right) T_{7}^{-3 / 2} F_{e 0} \mathrm{kV} \mathrm{cm}{ }^{-1}
\end{gathered}
$$

Note that this result depends only weakly (through $\lambda$ ) on the density of the thermal plasma.

At this point it is useful to have an estimate of the magnitude of $F_{e 0}$. Application of the collisional thick-target model to the observed hard X-ray emission from flares gives electron fluxes ranging from $10^{32}$ electrons s ${ }^{-1}$ to $10^{37}$ electrons s ${ }^{-1}$ (cf. Holman et al. 2003; Warmuth et al. 2009). Reliable measurements of hard X-ray footpoint sizes have been difficult to obtain, but estimates tend to fall in the $1^{\prime \prime}-10^{\prime \prime}$ range (cf. Dennis \& Pernak 2009). Taking a typical footpoint area to be $10^{17} \mathrm{~cm}^{2}, F_{e 0}$ is estimated to range from $10^{15}$ electrons $\mathrm{s}^{-1} \mathrm{~cm}^{-2}$ to $10^{20}$ electrons $\mathrm{s}^{-1} \mathrm{~cm}^{-2}$. If the accelerated electron current is filamented so that only a fraction of the observed footpoint area is emitting, this could be a lower limit on the range of electron flux density values.

We see from Equation 8 that if the initial return-current electric field operates on an electron over a distance of $10^{9} \mathrm{~cm}$ with $T=10 \mathrm{MK}$, an electron flux density $\sim 10^{19}$ electrons $\mathrm{s}^{-1} \mathrm{~cm}^{-2}$ or greater is required to diminish the electron's energy by $10 \mathrm{keV}$. This reduces to $\sim 10^{18}$ electrons $\mathrm{s}^{-1} \mathrm{~cm}^{-2}$ or greater if $T=2 \mathrm{MK}$.

An upper limit on $F_{e 0}$ can be estimated from the expectation that the number density of nonthermal electrons, $N_{b}$, should be much less than the number density of electrons in the ambient plasma, $n$. The number density of nonthermal electrons near the injection 
point, $N_{b 0}$, is $\int_{E_{c}}^{\infty}[F(E, 0) / v] d E$. Using Equation 5, this gives

$$
N_{b 0}=\left(\frac{\delta-1}{\delta-0.5}\right)\left(\frac{m_{e}}{2 E_{c}}\right)^{1 / 2} F_{e 0}=1.2 \times 10^{8}\left(\frac{\delta-1}{\delta-0.5}\right)\left(\frac{20}{E_{c}}\right)^{1 / 2}\left(\frac{F_{e 0}}{10^{18}}\right) \quad \mathrm{cm}^{-3} .
$$

A determination of the coronal plasma density in the impulsive phase of flares has been difficult to obtain. Flare coronal plasma densities as high as $10^{13} \mathrm{~cm}^{-3}$ have been inferred (e.g., Feldman et al. 1994; Phillips et al. 1996), generally for compact regions at times during or soon after the impulsive phase. Impulsive-phase coronal densities as high as $10^{12} \mathrm{~cm}^{-3}$ are likely. Requiring $N_{b 0}$ to be no higher than $10^{11} \mathrm{~cm}^{-3}$ gives an upper limit on $F_{e 0}$ of $10^{21}$ electrons s${ }^{-1} \mathrm{~cm}^{-2}$.

The return-current electric field strength decreases with distance as electrons are lost from the flux density distribution. An important insight is obtained upon recognizing that electrons are not lost from the distribution until their energy is reduced to $E_{t h}$. The lowest energy electrons are the first to be thermalized, those with energy $E_{c}$. The return-current electric field strength remains constant and equal to $E_{r c 0}$ until the distance $x_{r c}$ is reached at which those electrons with initial energy $E_{c}$ are thermalized and lost from the distribution. This occurs when $E_{t h}=E_{c}-e E_{r c 0} x_{r c}$, or when

$$
x_{r c}=\frac{E_{c}-E_{t h}}{e E_{r c 0}}=1.50 \times 10^{27} \frac{T_{7}^{3 / 2}\left(E_{c}-E_{t h}\right)}{(\lambda / 20) F_{e 0}} \mathrm{~cm},
$$

where the energies $E_{c}$ and $E_{t h}$ are in $\mathrm{keV}$. For $F_{e 0}=10^{19}$ electrons s${ }^{-1} \mathrm{~cm}^{-2}, T=10 \mathrm{MK}$, $E_{c}=20 \mathrm{keV}$, and $E_{t h}=2 \mathrm{keV}, x_{r c}=2.7 \times 10^{9} \mathrm{~cm}$, on the order of the half-length of a flare loop.

The return-current electric field strength $E_{r c}$ would not necessarily be constant below $x_{r c}$ if the injected electron flux density distribution does not have a sharp low-energy cutoff. For a flatter low-energy cutoff below some characteristic energy $E_{c}, x_{r c}$ becomes the distance at which $E_{r c}$ begins a more rapid decrease. 


\subsection{The Electron Distribution Function}

The evolution of the electron flux density distribution function with distance $x$ can be derived assuming conservation of electron flux density:

$$
F(E, x) d E=F\left(E_{0}, 0\right) d E_{0},
$$

where $E_{0}$ is the particle energy at $x=0$. The assumption of flux conservation seems incorrect, since electrons are lost from the distribution and, therefore, flux is not conserved. However, we can make this assumption as long as the loss of electron flux can be treated as a boundary condition. In our problem the boundary condition is that electrons are thermalized and removed from the distribution at $E=E_{t h}$. Above $E_{t h}$ flux is conserved.

Flux density is conserved only if the cross-sectional area of the electron beam (i.e., of the current carried by the accelerated electrons) remains constant. As long as any changes in cross-sectional area are independent of electron energy, such a change scales the magnitude of the flux density distribution function by $A(x) / A(0)$, where $A$ is the cross-sectional area of the beam. Since the focus here is on return-current losses, I assume that $A$ remains constant.

Rewriting Equation 11 as $F(E, x)=F\left(E_{0}, 0\right)\left(d E_{0} / d E\right)$, Equation 7 gives $d E_{0} / d E=1$ and $E_{0}=E+V(x) . F\left(E_{0}, 0\right)$ is given by Equation 5. Therefore, the distribution function at position $x$ is

$F(E, x)= \begin{cases}(\delta-1) E_{c}^{\delta-1} F_{e 0}(E+V(x))^{-\delta} \text { electrons s }{ }^{-1} \mathrm{~cm}^{-2} \mathrm{keV}^{-1} & E \geq E_{c}-V(x) \\ 0 & E<E_{c}-V(x) .\end{cases}$

The original sharp low-energy cutoff moves downward in energy with increasing $x$ until $V(x)=E_{c}$ or, more correctly, accounting for our boundary condition, until $V(x)=E_{c}-E_{t h}$.

Once $V(x)=E_{c}-E_{t h}$, the inequalities on the right side of Equation 12 become $E \geq E_{t h}$ 
and $E<E_{t h}$. The potential drop $V(x)$ becomes a new, effective low-energy cutoff, with the distribution function becoming flat for $E \ll V(x)$. If $F\left(E_{0}, 0\right)$ contained a high-energy cutoff or break, this break energy would evolve with distance as $E_{\text {break }}(x)=E_{\text {break }}(0)-V(x)$. Since $V$ acts as a cutoff energy to the electron distribution function, I will sometimes write its units as $\mathrm{keV}$ instead of $\mathrm{kV}$.

The remaining challenge is to determine the functional form of $V(x)$. This is most easily accomplished by recognizing that

$$
\frac{d V}{d x}=e E_{r c}(x)=e^{2} \eta F_{e}(x)
$$

where

$$
F_{e}(x)=\int_{0}^{\infty} F(E, x) d E .
$$

Integrating Equation 12 over $E$ gives $F_{e}(x)$ :

$$
F_{e}(x)=\left\{\begin{array}{lr}
F_{e 0} & V(x) \leq E_{c}-E_{t h} \\
E_{c}^{\delta-1} F_{e 0}\left(E_{t h}+V(x)\right)^{1-\delta} \text { electrons s}{ }^{-1} \mathrm{~cm}^{-2} & V(x)>E_{c}-E_{t h} .
\end{array}\right.
$$

The inequalities on the right side of Equation 15 are equivalent to $x \leq x_{r c}$ and $x>x_{r c}$.

This result for the electron flux density can now be used in Equation 13 to solve for $V(x)$. For simplicity, I will assume here that $\eta$ (primarily $T$ ) has no spatial dependence and, therefore, is constant. A spatially dependent resistivity is discussed in Section 2.3. The spatial dependence of the potential drop is thus found to be, after some algebraic manipulation,

$$
V(x)= \begin{cases}e E_{r c 0} x=e^{2} \eta F_{e 0} x \quad \text { statvolt } & x \leq x_{r c} \\ E_{c}\left[\delta\left(\frac{e E_{r c 0}}{E_{c}}\right)\left(x-x_{r c}\right)+1\right]^{1 / \delta}-E_{t h} & x>x_{r c} .\end{cases}
$$

The dependence on $x$ of $F(E, x), F_{e}(x), E_{r c}(x)\left(=\eta e F_{e}(x)\right)$, and $V(x)$ is now fully determined. The potential drop increases linearly with $x$ for $x \leq x_{r c}$ since, as discussed 
above, $E_{r c}$ is constant below $x_{r c}$. Beyond $x_{r c}, E_{r c}$ is decreasing and, for $x \gg x_{r c}, V(x)$ increases slowly as $x^{1 / \delta}$. While $F_{e}$ and $E_{r c}$ are constant below $x_{r c}$, they fall off as $x^{-(1-1 / \delta)}$ for $x \gg x_{r c}$.

$F_{e}$ and $E_{r c}$ are plotted as a function of distance in Figure 1 for $\delta=5$, a fixed plasma temperature of $10^{7} \mathrm{~K}$, and several values of $F_{e 0}$. The value of $\lambda$ is fixed at 20. This spatial dependence is also shown for $\delta=3$ and $\delta=7$ when $F_{e 0}=10^{21}$ electrons s $\mathrm{cm}^{-2}$. The initial low-energy cutoff and thermalization energy are taken to be $E_{c}=20 \mathrm{keV}$ and $E_{t h}=2.5 k T=2.15 \mathrm{keV}$. The point at which the curves begin to decrease determines the value of $x_{r c}\left(x_{r c}=2.7 \times 10^{9} \mathrm{~cm}\right.$ when $F_{e 0}=10^{19}$ electrons s $\left.\mathrm{s}^{-1} \mathrm{~cm}^{-2}\right)$. Corresponding curves for the potential drop $V(x)$ are shown in Figure 2. Here the distance $x_{r c}$ is located where the curves cross the level $V=E_{c}-E_{t h}$. There is no dependence on $\delta$ until $x>x_{r c}$. Above $x_{r c}, F_{e}$ and $E_{r c}$ decrease more rapidly with $x$ as $\delta$ increases and $V$ increases less rapidly with $x$ as $\delta$ increases.

The solution obtained here agrees with that of Knight \& Sturrock (1977) if we set $E_{t h}=0$ and account for the difference in the assumed form of the injected electron flux density distribution function. Instead of a sharp low-energy cutoff, Knight \& Sturrock assume an injected distribution function of the form $F(E, 0)=K\left(E+E_{0}\right)^{-\delta}$, a distribution that already becomes flat below the initial cutoff energy $E_{0}$.

\subsection{Column Resistivity and Resistance}

In this section I generalize the previous results to include spatial dependence of the plasma resistivity. Just as collisional losses depend fundamentally on the column density $N(x)=\int_{0}^{x} n\left(x^{\prime}\right) d x^{\prime}$, return-current losses depend on the column resistivity, 
$\rho(x)=\int_{0}^{x} \eta\left(x^{\prime}\right) d x^{\prime}$. Equation 13 can then be rewritten as

$$
\frac{d V}{d \rho}=e^{2} F_{e}(V)
$$

and Equation 16 becomes

$$
V(\rho)= \begin{cases}e^{2} F_{e 0} \rho \text { statvolt } & \rho \leq \rho_{r c} \\ E_{c}\left[\delta\left(\frac{e^{2} F_{e 0}}{E_{c}}\right)\left(\rho-\rho_{r c}\right)+1\right]^{1 / \delta}-E_{t h} & \rho>\rho_{r c}\end{cases}
$$

This equation contains a generalization of the return-current losses critical distance $x_{r c}$ to the critical column resistivity $\rho_{r c}$, given by

$$
\rho_{r c}=\frac{E_{c}-E_{t h}}{e^{2} F_{e 0}}=6.94 \times 10^{9} \frac{\left(E_{c}-E_{t h}\right)}{F_{e 0}} \mathrm{~cm} \mathrm{~s},
$$

where, as in Equation 10, $E_{c}$ and $E_{t h}$ are in keV. For the same values of $E_{c}, E_{t h}$, and $F_{e 0}$ as in Equation 10, $\rho_{r c}=1.25 \times 10^{-8} \mathrm{~cm} \mathrm{~s}$.

If the resistivity is classical (Equation 3) and we ignore the weak dependence of $\lambda$ on $x$, we can write $T(x)=T_{0} \hat{T}(x)$, giving $\eta(x)=\eta_{0} \hat{T}(x)^{-3 / 2}$. The column resistivity then becomes $\rho=\eta_{0} \int_{0}^{x}\left[\hat{T}\left(x^{\prime}\right)\right]^{-3 / 2} d x^{\prime}$. Note that if $E_{t h}$ has a significant dependence on $x$, Equation 17 (or Equation 13) is no longer separable and must be solved numerically.

The column resistivity is related to the total resistance at position $x$, given by $R(x)=V(x) / I(x)=V(x) /\left(e F_{e}(x) A\right)$, where $I(x)=J(x) A$ is the total current at $x$ and $A$ is the area of the electron beam (and return current). For $\rho \leq \rho_{r c}, R=\rho / A$. At $\rho_{r c}$,

$$
R_{r c}=\frac{\rho_{r c}}{A}=6.94 \times 10^{9} \frac{\left(E_{c}-E_{t h}\right)}{F_{e 0} A} \mathrm{~s} \mathrm{~cm}^{-1}=6.24 \times 10^{21} \frac{\left(E_{c}-E_{t h}\right)}{F_{e 0} A} \mathrm{ohm} .
$$

Using the same values as above and $A=10^{16} \mathrm{~cm}^{2}, R_{r c}$ is only $1.12 \times 10^{-12} \mathrm{ohm}$. The corresponding current $I_{r c}$ is $4.8 \times 10^{25}$ statampere, or $1.6 \times 10^{16}$ ampere. 


\subsection{Return-Current Plasma Heating}

The heating rate per unit volume, $Q(x) \mathrm{erg} \mathrm{s}^{-1} \mathrm{~cm}^{-3}$, of the thermal plasma resulting from return-current losses is given by the spatial rate of change of the energy flux density of the accelerated electrons:

$$
Q(x)=\frac{d}{d x} \int F(E, x) E d E=\int \frac{d F(E, x)}{d x} E d E=\int \frac{d F(E, x)}{d E} \frac{d E}{d x} E d E .
$$

The last term suggests that when more than one energy loss mechanism is operating and the total energy losses can be written as the sum of the losses from each mechanism, the total heating rate can be obtained by simply summing the heating rate for each mechanism. This is misleading, however, since the electron flux density distribution function, $F(E, x)$, is changed by the loss mechanisms.

Substituting Equations 6 and 8 (generalized to all positions $x$ ) for return current losses into Equation 21 gives

$$
Q(x)=-e E_{r c}(x) \int \frac{d F(E, x)}{d E} E d E=-e^{2} \eta F_{e}(x) \int \frac{d F(E, x)}{d E} E d E .
$$

The minimum value of the lower limit to the integral is $E_{t h}$ and the upper limit is infinity. Integrating by parts and using the requirement that $E F(E, x)$ goes to zero at $E=\infty$ and the definition of $F_{e}(x)$ (Equation 14) gives

$$
Q(x)=\eta e^{2}\left[F_{e}(x)\right]^{2}+\eta e^{2} F_{e}(x) E_{t h} F\left(E_{t h}, x\right) .
$$

The first term in Equation 23 is Joule heating by the return current, $J_{r c} E_{r c}$. The last term is heating by the nonthermal electrons when their energy is reduced to $E_{t h}$ and they are thermalized. For the sharp low-energy cutoff, this term does not contribute until $V(x) \geq E_{c}-E_{t h}$ or, equivalently, until $x \geq x_{r c}$. This gives a discontinuity at $x_{r c}$, resulting from the simplifying assumption that the electrons become thermalized at the discrete energy $E_{t h}$. 
Substituting Equations 12 and 15 into Equation 23, the volumetric heating rate becomes

$$
Q(x)= \begin{cases}\eta e^{2} F_{e 0}^{2} \quad \operatorname{erg~s}^{-1} \mathrm{~cm}^{-3} & x<x_{r c} \\ \eta e^{2} F_{e 0}^{2}\left(\delta \frac{E_{t h}}{E_{c}}+\frac{V(x)}{E_{c}}\right)\left(\frac{E_{t h}}{E_{c}}+\frac{V(x)}{E_{c}}\right)^{1-2 \delta} & x \geq x_{r c} .\end{cases}
$$

The two solid curves in Figure 3 show this volumetric heating rate as a function of distance for $E_{t h}=2.5 k T=2.15 \mathrm{keV}$ and $E_{t h}=18 \mathrm{keV}$, respectively, with $F_{e 0}=10^{19}$ electrons s ${ }^{-1}$ $\mathrm{cm}^{-2}, T=10 \mathrm{MK}, \lambda=20, E_{c}=20 \mathrm{keV}$, and $\delta=5$. The results for $E_{t h}=18 \mathrm{keV}$ are reduced by a factor of 100 to avoid overlapping curves. The vertical dashed lines show the value of $x_{r c}$ for the two values of $E_{t h}$. The dashed curves show the heating rate for Joule heating alone (first term in Equation 23). The Joule heating rate (as well as the total heating rate) is constant below $x_{r c}$. At high values of $x\left(x \gg x_{r c}\right)$ the Joule heating rate falls off as $x^{\frac{2}{\delta}-2}$ if $T$ (and $\lambda$ ) has no spatial dependence.

The dotted curves in Figure 3 show the contribution of electron thermalization (last term in Equation 23) to the total volumetric heating rate. This term is negligible when $E_{t h} \ll E_{c}$. For $E_{t h}=2.15 \mathrm{keV}$, Figure 3 shows a small, but significant, contribution from this term. Electron thermalization can exceed Joule heating above $x_{r c}$, however, when $E_{t h}$ is comparable to $E_{c}$, as is the case for $E_{t h}=18 \mathrm{keV}$. The discontinuity in the total heating rate at $x=x_{r c}$ results from our assumption that the electrons are thermalized at the discrete energy $E_{t h}$ (assumption 6 in Section 2). This discontinuity would be smoothed out in a more realistic model. When $x \gg x_{r c}$ this term falls off somewhat faster, as $x^{\frac{1}{\delta}-2}$, than the Joule heating term. The total return-current volumetric heating rate falls off as $x^{\frac{2}{\delta}-2}$ when $x \gg x_{r c}$.

We can estimate the electron flux density required for the volumetric heating to be significant in the upper, coronal part of the flare loop using the requirement $Q\left(x<x_{r c}\right) \Delta t \gtrsim n k T$ and Equation 24, where $\Delta t$ is the interval of time over which the

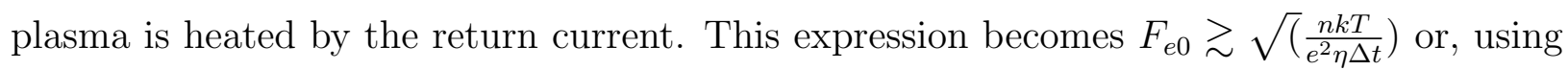


Equation 3,

$$
F_{e 0} \gtrsim 6.4 \times 10^{16}\left(\frac{20}{\lambda} \frac{n_{9}}{\Delta t}\right)^{1 / 2} T_{6}^{5 / 4} \quad \text { electrons } \mathrm{s}^{-1} \mathrm{~cm}^{-2},
$$

where $\Delta t$ is in seconds. This result indicates that for a loop with typical active region temperature and density, an electron flux density $\sim 10^{17}$ electrons $\mathrm{s}^{-1} \mathrm{~cm}^{-2}$ is required. If the loop is already at a typical flare temperature, the electron flux density needs to be an order of magnitude higher.

As shown in Equation 24, the volumetric heating rate where $x<x_{r c}$ varies with the injected electron flux density as $F_{e 0}^{2}$. The dependence is much weaker when $x \gg x_{r c}$, with $Q \propto F_{e 0}^{\frac{2}{\delta}}$. The total heating rate $H\left(<x_{r c}\right)\left(\operatorname{erg~s}^{-1}\right)$ at distances less than $\mathrm{x}_{r c}$ is easily calculated, using equations 24 and 10, to be

$$
H\left(<x_{r c}\right)=F_{e 0}\left(E_{c}-E_{t h}\right) A=3.2 \times 10^{26}\left(\frac{F_{e 0}}{10^{18}}\right)\left(\frac{E_{c}-E_{t h}}{20}\right) A_{16} \quad \mathrm{erg} \mathrm{s}^{-1},
$$

where $A_{16}$ is the area of the electron beam (and return current) in units of $10^{16} \mathrm{~cm}^{2}$. It is interesting to compare this to the injected energy flux of the electron beam. The energy flux density of the injected electrons is

$$
P_{e 0}=\left(\frac{\delta-1}{\delta-2}\right) E_{c} F_{e 0}=3.20 \times 10^{10}\left(\frac{\delta-1}{\delta-2}\right)\left(\frac{E_{c}}{20}\right)\left(\frac{F_{e 0}}{10^{18}}\right) \quad \mathrm{erg} \mathrm{s}^{-1} \mathrm{~cm}^{-2} .
$$

From this we find that the ratio of the heating rate in the region $x<x_{r c}$ to the injected electron energy flux is

$$
\frac{H\left(<x_{r c}\right)}{P_{e 0} A}=\left(\frac{\delta-2}{\delta-1}\right)\left(1-\frac{E_{t h}}{E_{c}}\right)
$$

Thus, the fraction of the beam energy deposited in this region is greatest for a steep electron distribution and $E_{t h} \ll E_{c}$. However, when $E_{t h} \sim E_{c}$, as shown in Figure 3, the heat deposition is enhanced just beyond $x_{r c}$. For the case in Figure 3 with $E_{t h}=2.15 \mathrm{keV}$, the ratio of the heating rate at $x<x_{r c}$ to the injected electron energy flux is 0.67 . 


\subsection{Comparison of Return-Current and Collisional Energy Losses}

The energy lost by an electron per unit distance due to return-current losses is, from Equation 6 or Equation 13,

$$
\begin{aligned}
\left(\frac{d E}{d x}\right)_{r c}=-\frac{d V}{d x} & =-e^{2} \eta F_{e}(x)=-1.07 \times 10^{-17}\left(\frac{\lambda}{20}\right) T_{7}^{-3 / 2}\left(\frac{F_{e}}{10^{19}}\right) \quad \mathrm{erg} \mathrm{cm}^{-1} \\
& =-0.668\left(\frac{\lambda}{20}\right) T_{7}^{-3 / 2}\left(\frac{F_{e}}{10^{19}}\right) \quad \mathrm{keV} \mathrm{Mm}^{-1} .
\end{aligned}
$$

The energy lost per unit distance due to collisional losses is, from Equations 2.2 and 2.3 of Holman et al. (2011),

$$
\begin{gathered}
\left(\frac{d E}{d x}\right)_{\text {coll }}=-K \frac{n}{E}=-4.80 \times 10^{-27}\left(\frac{\Lambda}{23}\right) \frac{n}{E}=-2.40 \times 10^{-18}\left(\frac{\Lambda}{23}\right) n_{10}\left(\frac{20}{E}\right) \quad \mathrm{erg} \mathrm{cm}^{-1} \\
=-0.150\left(\frac{\Lambda}{23}\right) n_{10}\left(\frac{20}{E}\right) \quad \mathrm{keV} \mathrm{Mm}^{-1} .
\end{gathered}
$$

Here $\Lambda$ is the Coulomb logarithm for the interaction of a high-energy electron with thermal plasma and $E$ is in $\mathrm{keV}$. One $\mathrm{Mm}\left(=10^{8} \mathrm{~cm}\right)$ is approximately $1^{\prime \prime} .38$ at a distance of 1 AU. Therefore, the ratio of the return-current loss rate to the collisional loss rate is

$$
\frac{(d E / d x)_{r c}}{(d E / d x)_{\text {coll }}}=4.45\left(\frac{\lambda}{20}\right)\left(\frac{23}{\Lambda}\right) T_{7}^{-3 / 2} n_{10}^{-1}\left(\frac{E}{20}\right)\left(\frac{F_{e}}{10^{19}}\right) .
$$

From this result we see that the return-current loss rate exceeds the collisional loss rate at $20 \mathrm{keV}$ when $F_{e} \gtrsim 2.2 \times 10^{18} n_{10} T_{7}^{3 / 2}$ electrons s${ }^{-1} \mathrm{~cm}^{-2}$. Return-current losses will dominate at even lower electron beam flux densities when the ambient plasma density and/or temperature is lower. On the other hand, the return-current losses (when $x>x_{r c}$ or, more generally, $\rho>\rho_{r c}$ ) and collisional losses reduce the value of $F_{e}$ with increasing distance.

In Figure 4 return-current energy losses as a function of distance $x$ are plotted for electrons with four initial energies, $E_{0}=20,30,40$ and $50 \mathrm{keV}$ (solid curves), and compared with collisional energy losses for two values of the plasma density, $n=10^{10} \mathrm{~cm}^{-3}$ (dashed 
curves) and $n=10^{11} \mathrm{~cm}^{-3}$ (dotted curves). The return-current losses are for $F_{e 0}=7 \times 10^{18}$ electrons $\mathrm{s}^{-1} \mathrm{~cm}^{-2}\left(P_{e 0}=3 \times 10^{11} \mathrm{erg} \mathrm{s}^{-1} \mathrm{~cm}^{-2}\right), T=10 \mathrm{MK}, \lambda=20, \delta=5, E_{c}=20 \mathrm{keV}$, and $E_{t h}=2.5 k T=2.15 \mathrm{keV}$. The vertical dashed line shows the value of $x_{r c}(38 \mathrm{Mm})$. The return-current energy loss curves are computed using Equations 7 and 16. The collisional energy loss curves are computed using the solution to Equation 30, $E(x)=\sqrt{E_{0}^{2}-2 K n x}$.

For all initial electron energies in Figure 4, return-current losses exceed collisional losses when $n=10^{10} \mathrm{~cm}^{-3}$, and collisional losses exceed return-current losses when $n=10^{11} \mathrm{~cm}^{-3}$, consistent with results obtained from Equation 31. The return-current energy losses are seen to slow at distances greater than $x_{r c}$, when the electron flux density and, therefore, the electric field strength begins to decrease. Even when the return-current energy losses dominate below $x_{r c}$, collisional energy losses can dominate at greater distance because of this reduction in the rate of energy loss with distance.

The return-current energy loss rate is further slowed by collisional losses when $x>x_{\text {coll }} \equiv\left(E_{c}^{2}-E_{t h}^{2}\right) / 2 K n \simeq 0.17\left(E_{c}^{2}-E_{t h}^{2}\right) / n_{10}$ Mm or, more generally, when the column density $N>N_{\text {coll }} \equiv\left(E_{c}^{2}-E_{t h}^{2}\right) / 2 K \simeq 1.7 \times 10^{17}\left(E_{c}^{2}-E_{t h}^{2}\right) \mathrm{cm}^{-2}$, a rate reduction not reflected in Figure 4. The distance at which the electron flux density begins to decrease will in fact be determined by $x_{\text {coll }}$ if $x_{\text {coll }}<x_{r c}$. This inequality can be written as $n>n_{\text {coll }}$, where

$$
n_{\text {coll }}=\frac{e^{2} \eta F_{e 0}}{2 K}\left(E_{c}+E_{t h}\right)=2.23 \times 10^{10}\left(\frac{\lambda}{20}\right)\left(\frac{23}{\Lambda}\right) T_{7}^{-3 / 2}\left(\frac{F_{e 0}}{10^{19}}\right)\left(\frac{E_{c}+E_{t h}}{20}\right) \mathrm{cm}^{-3} .
$$

For the parameters used in Figure $4, x_{\text {coll }}<x_{r c}$ for the dotted curves, when $n=10^{11} \mathrm{~cm}^{-3}$ $\left(n_{\text {coll }}=1.7 \times 10^{10} \mathrm{~cm}^{-3}\right)$. Therefore, when $n=10^{11} \mathrm{~cm}^{-3}$ and the loss of electrons due to collisions is included, the return-current loss rate will begin to decrease beyond $x_{\text {coll }} \simeq 6.7 \mathrm{Mm}$ instead of $x_{r c}$ and the return-current energy losses beyond $x_{\text {coll }}$ are less than the results shown in Figure 4. 


\subsection{X-Ray Source Brightness and Return-Current Losses}

The bremsstrahlung X-rays from the nonthermal electrons accelerated in flares is usually observed as thick-target emission from compact regions at the footpoints of flare magnetic loops. The thickness of these regions is usually small compared to the height of the flare loops, because of the rapid downward increase in plasma density in the relatively thin transition region and chromosphere. Therefore, we can define a distance from the electron injection region at $x=0$ to the thick-target $\mathrm{X}$-ray emission region, $x_{t t}$. From hard X-ray time delay studies, Aschwanden et al. (1996) have found this distance to range from $6-60 \mathrm{Mm}$. A single value of $x_{t t}$ is a good approximation as long as the thickness of the thick-target source region is small compared to the value of $x_{t t}$. The value of $x_{t t}$ becomes significantly dependent on electron energy if the corona becomes collisionally thick to electron energies of interest, as might occur during chromospheric evaporation.

In general, X-rays of energy $\epsilon$ and below are affected by return-current losses when $V\left(x_{t t}\right) \sim \epsilon$. The electron flux density entering into the thick-target region is not diminished by return-current losses until $V\left(x_{t t}\right)>E_{c}-E_{t h}$ or, equivalently, until $x_{r c}<x_{t t}$ (Equation 15). We can use this condition as an estimate of the minimum requirement for the X-ray emission to be affected, although the lower end of the electron distribution function is diminished somewhat before $V\left(x_{t t}\right)>E_{c}-E_{t h}$ (Equation 12). Noting the dependence of $x_{r c}$ on $F_{e 0}$, we can derive a critical injected electron flux density above which the flux density into the thick-target region begins to diminish, $F_{e 0}^{t t}$. Using Equation 10, we obtain

$$
F_{e 0}^{t t}=\frac{E_{c}-E_{t h}}{e^{2} \eta x_{t t}}=1.0 \times 10^{19}\left(\frac{20}{\lambda}\right) T_{7}^{3 / 2}\left(\frac{3 \times 10^{9}}{x_{t t}}\right)\left(\frac{E_{c}-E_{t h}}{20}\right) \text { electrons } \mathrm{s}^{-1} \mathrm{~cm}^{-2} .
$$

This result indicates that the injected electron flux density for which return-current losses become significant is on the order of $10^{18}-10^{19}$ electrons $\mathrm{s}^{-1} \mathrm{~cm}^{-2}$. In terms of electron energy flux density (Equation 27), this corresponds to $4 \times 10^{10}-4 \times 10^{11} \mathrm{erg} \mathrm{s}^{-1} \mathrm{~cm}^{-2}$ for $E_{c}=20 \mathrm{keV}$ and $\delta=5$. It is interesting that these injected energy flux densities are on the 
order of those for which the transition from gentile to explosive chromospheric evaporation is estimated to occur (Fisher et al. 1985).

The dependence of $F_{e}\left(x_{t t}\right)$ on $F_{e 0}$ is plotted in Figure 5, using Equations 15 and 16 , for $x_{t t}=30 \mathrm{Mm}, T=10 \mathrm{MK}, \lambda=20, \delta=4, E_{c}=20 \mathrm{keV}$, and two values of $E_{t h}$, $E_{t h}=2.5 k T=2.15 \mathrm{keV}$ (solid curve) and $E_{t h}=18 \mathrm{keV}$ (long dashes). The dotted vertical lines show the value of $F_{e 0}^{t t}$ for both cases. It is interesting that for $E_{t h}=2.15 \mathrm{keV}$, as $F_{e 0}$ increases a local maximum is reached at $F_{e 0}^{t t}$ followed by a local minimum and then $F_{e}\left(x_{t t}\right)$ continues to increase after the local minimum. For $E_{t h}=18 \mathrm{keV} F_{e}\left(x_{t t}\right)$ only increases with increasing $F_{e 0}$, but at a much lower rate. Both curves increase as $F_{e 0}^{1 / \delta}$ well above $F_{e 0}^{t t}$ and the local minimum. We see that the increase of $F_{e}\left(x_{t t}\right)$ is so slow that it is effectively limited to values on the order of $F_{e 0}^{t t}$, especially when $\delta$ is large and the local minimum is present.

An analytical search for extrema in $F_{e}(x)$ as a function of $F_{e 0}$ shows that the local maximum and corresponding minimum are obtained when $E_{t h}<\frac{\delta-2}{\delta-1} E_{c}$. The value of $F_{e}(x)$ at the local minimum is

$$
F_{e}^{m i n}(x)=\frac{E_{c}}{e^{2} \eta x}(\delta-1)^{\frac{1}{\delta}-1}\left[\delta\left(1-\frac{E_{t h}}{E_{c}}\right)-1\right]^{\frac{1}{\delta}} .
$$

The value of $F_{e 0}$ at which this minimum occurs is

$$
F_{e 0}=\frac{E_{c}}{e^{2} \eta x}\left[\delta\left(1-\frac{E_{t h}}{E_{c}}\right)-1\right] .
$$

The value of $F_{e}^{\text {min }}\left(x_{t t}\right)$ and the corresponding value of $F_{e 0}$ are indicated in Figure 5 by the crossed short-dashed lines. The dotted curve in Figure 5 shows another result without a local minimum. This curve is for $\delta=2.1$ and $E_{t h}=2.15 \mathrm{keV}$. This curve is close to the maximum rate at which $F_{e}\left(x_{t t}\right)$ can increase with $F_{e 0}$ beyond $F_{e 0}^{t t}$.

The brightness spectrum of the X-rays emitted from the thick-target source region by electrons experiencing energy losses at the rate $d E / d t$ is given by (Brown 1971; Emslie 
1980; Holman 2003; Holman et al. 2011)

$$
B(\epsilon)=\frac{1}{4 \pi R^{2}} \int_{\epsilon}^{\infty} F\left(E_{0}\right) \int_{E_{0}}^{\epsilon} \frac{n_{i}(x) \sigma(\epsilon, E)}{d E / d x} d E d E_{0},
$$

where $R$ is the distance from the observer to the source region, usually one astronomical unit, $n_{i}$ is the plasma ion number density, and $\sigma(\epsilon, E)$ is the bremsstrahlung cross section. $F\left(E_{0}\right)$ is the injected electron flux density distribution. The inner integral is the photon flux distribution above energy $\epsilon$ (and below photon energy $E_{0}$ ) emitted by a single electron of energy $E_{0}$. The outer integral integrates this result over the injected distribution of all electrons with energies $E_{0} \geq \epsilon$. If $\epsilon<E_{c}$, the lower limit of the outer integral becomes $E_{c}$. The X-ray brightness spectrum rather than the usual flux spectrum is obtained because $F\left(E_{0}\right)$ is the electron flux density distribution rather than the flux distribution (electrons $\left.\mathrm{s}^{-1} \mathrm{keV}^{-1}\right)$.

If we continue with our assumption that return-current losses are not significant in the thick-target region and use the Kramers approximation to the bremsstrahlung cross section, a simple analytic result is obtained for the X-ray brightness spectrum. In this case $d E / d x$ is the result for collisional losses alone (Equation 30) and $F\left(E_{0}\right)$ is given by Equation 12 at $x_{t t}$. The Kramers cross section is $\sigma(\epsilon, E)=\overline{Z^{2}} \sigma_{0} /(\epsilon E)$, where $\sigma_{0}=7.90 \times 10^{-25} \mathrm{~cm}^{2} \mathrm{keV}$ and $\overline{Z^{2}} \simeq 1.4$ is the weighted mean square atomic number of the target plasma, weighted by the number density of each ion species in the plasma. From Equation 36, the X-ray brightness spectrum (for $\epsilon \geq \max \left[E_{c}-V\left(x_{t t}\right), E_{t h}\right]$ and for $R=1 \mathrm{AU}$ ) is

$$
B(\epsilon) \approx 1.17 \times 10^{-34} \frac{E_{c}^{\delta-1} F_{e 0}}{\delta-2} \epsilon^{-1}\left(\epsilon+V\left(x_{t t}\right)\right)^{2-\delta} \text { photons } \mathrm{cm}^{-2} \mathrm{~s}^{-1} \mathrm{~cm}^{-2} \mathrm{keV}^{-1}
$$

This has the expected $\epsilon^{-(\delta-1)}$ dependence when $\epsilon \gg V\left(x_{t t}\right)$. It also has the expected $\epsilon^{-1}$ dependence when $\epsilon \ll V\left(x_{t t}\right)$.

Emslie (1980) estimated that in the presence of return-current losses the integrated bremsstrahlung photon brightness above $20 \mathrm{keV}$ will be limited to $10^{-15}$ photons $\mathrm{cm}^{-2} \mathrm{~s}^{-1}$ 
$\mathrm{cm}^{-2}$. He estimated that this limiting brightness will be reached when the injected electron flux density (above $20 \mathrm{keV}$ ) exceeds $10^{19}$ electrons $\mathrm{cm}^{-2} \mathrm{~s}^{-1}$. This estimate was obtained by arguing that when return-current losses are large enough to dominate over collisional losses, the integrand for computing the thick-target bremsstrahlung X-ray emission becomes independent of the injected electron beam flux density. Here I show that this is not an upper limit, but instead is roughly the integrated X-ray brightness when the electron flux density and, consequently, the X-ray brightness begin to increase more slowly than linearly as the injected electron flux density increases. The results derived here show how the estimates scale with other physical parameters.

It is interesting to first look at the dependence of the integral of Equation 37 over all photon energies above $20 \mathrm{keV}, B(>20 \mathrm{keV})$, on the injected electron flux density $F_{e 0}$, even though this result assumes that return-current losses do not dominate in the thick-target region. The leading term gives a linear dependence on $F_{e 0}$, but $V\left(x_{t t}\right)$ also depends on $F_{e 0}$. Results obtained by numerically integrating Equation 37 are shown in Figure 6 for $x_{t t}=10 \mathrm{Mm}, T=10 \mathrm{MK}, \lambda=20, E_{c}=20 \mathrm{keV}, E_{t h}=2.15 \mathrm{keV}$, and five values of $\delta$. We see that there is no maximum value $\sim 10^{-15}$ photons $\mathrm{cm}^{-2} \mathrm{~s}^{-1} \mathrm{~cm}^{-2}$ or higher, but the rate of increase of the integrated X-ray brightness with $F_{e 0}$ does decrease as $F_{e 0}$ approaches the value of $F_{e 0}^{t t}\left(\right.$ as $V\left(x_{t t}\right)$ approaches $\left.20 \mathrm{kV}\right)$. For values of $\delta \gtrsim 5, B(>20 \mathrm{keV})$ goes through a local maximum followed by a local minimum before continuing to rise at a reduced rate, similar to the evolution of $F_{e}\left(x_{t t}\right)$ with $F_{e 0}$ (Figure 5). The rate of increase of $B(>20 \mathrm{keV})$ with $F_{e 0}$ first decreases and then increases to the new, lower rate, as $F_{e 0}$ exceeds $F_{e 0}^{t t}$ and the electron flux density at $x_{t t}$ begins to decrease below the value of $F_{e 0}$.

Let's now consider the assumption that return-current losses dominate everywhere, so $d E / d x$ is given by Equation 29. Since return-current losses are proportional to $F_{e}(x)$ and $F_{e}(x)$ becomes arbitrarily small as $x$ increases (Equation 15), return-current losses cannot 
be everywhere smaller than collisional losses, as assumed. Nevertheless, we can formally explore the consequences of this assumption. The dependence on $F_{e 0}$ can best be seen by making the potential drop $V$ the integration variable. The inner integral in Equation 36 becomes, using Equations 7 and 29,

$$
\nu\left(\epsilon, E_{0}\right)=\int_{0}^{E_{0}-\epsilon} \sigma\left(\epsilon, E_{0}-V\right) \frac{n_{i}(x(V))}{e^{2} \eta(x(V)) F_{e}(V)} d V .
$$

The dependence of $n_{i}$ and $\eta$ on $V$ is obtained by solving Equation 18 for $\rho$, and then for $x$. Substituting Equation 15 for $F_{e}(V)$ gives

$$
\nu\left(\epsilon, E_{0}\right)=\frac{1}{e^{2} F_{e 0}} \times\left\{\begin{array}{lr}
\int_{0}^{E_{0}-\epsilon} \sigma\left(\epsilon, E_{0}-V\right) \frac{n_{i}(x(V))}{\eta(x(V))} d V & V \leq E_{c}-E_{t h} \\
\int_{0}^{E_{c}-E_{t h}} \sigma\left(\epsilon, E_{0}-V\right) \frac{n_{i}(x(V))}{\eta(x(V))} d V & \\
+E_{c}^{1-\delta} \int_{E_{c}-E_{t h}}^{E_{0}-\epsilon} \sigma\left(\epsilon, E_{0}-V\right) \frac{n_{i}(x(V))}{\eta(x(V))}\left(E_{t h}+V\right)^{\delta-1} d V & V>E_{c}-E_{t h}
\end{array}\right.
$$

As noted by Emslie (1980), the linear dependence on $F_{e 0}$ cancels out, since $F\left(E_{0}\right)$ in the outer integral is proportional to $F_{e 0}$. The X-ray brightness still depends on $F_{e 0}$ through $n_{i}(x(V)) / \eta(x(V))$, however, since the relationship between $V$ and $x$ depends on $F_{e 0}$. Therefore, the fact that these leading terms cancel out does not lead to a clear upper limit, independent of $F_{e 0}$. However, it is interesting that if $n_{i} / \eta$ has no spatial dependence, $B(\epsilon)$ is independent of $F_{e 0}$. Therefore, although unrealistic, it is instructive to consider this case next.

We again estimate the integrated X-ray brightness using the Kramers bremsstrahlung cross section. Since we are interested in $B(>\epsilon)$, we use the integrated cross section for all photons emitted by an electron of energy $E$ above photon energy $\epsilon$ : $\sigma(>\epsilon, E)=$ $\overline{Z^{2}} \sigma_{0} \ln (E / \epsilon) / E$. For constant $n_{i} / \eta$, the integrated brightness becomes (Equations 36 and 39, together with Equation 3)

$$
B(>\epsilon)=\frac{\sigma_{0} / e^{2}}{4 \pi R^{2}}\left(\frac{n_{i}}{\eta}\right) f\left(\frac{\epsilon}{E_{c}}, \delta, \frac{E_{t h}}{E_{c}}\right)
$$




$$
\simeq 4.21 \times 10^{-15} n_{10} T_{7}^{3 / 2} f\left(\frac{\epsilon}{E_{c}}, \delta, \frac{E_{t h}}{E_{c}}\right) \quad \text { photons } \mathrm{cm}^{-2} \mathrm{~s}^{-1} \mathrm{~cm}^{-2}
$$

where, writing $\hat{\epsilon}=\epsilon / E_{c}, \hat{E}_{t h}=E_{t h} / E_{c}, \hat{E}_{0}=E_{0} / E_{c}$, and $\hat{V}=V / E_{c}$,

$$
\begin{aligned}
& \frac{f\left(\hat{\epsilon}, \delta, \hat{E}_{t h}\right)}{\delta-1}= \int_{\hat{\epsilon}}^{1-\hat{E}_{t h}+\hat{\epsilon}} \hat{E}_{0}^{-\delta} \int_{0}^{\hat{E}_{0}-\hat{\epsilon}} \frac{\ln \left(\left(\hat{E}_{0}-\hat{V}\right) / \hat{\epsilon}\right)}{\hat{E}_{0}-\hat{V}} d \hat{V} d \hat{E}_{0}+ \\
& \int_{1-\hat{E}_{t h}+\hat{\epsilon}}^{\infty} \hat{E}_{0}^{-\delta} \int_{0}^{1-\hat{E}_{t h}} \frac{\ln \left(\left(\hat{E}_{0}-\hat{V}\right) / \hat{\epsilon}\right)}{\hat{E}_{0}-\hat{V}} d \hat{V} d \hat{E}_{0}+ \\
& \int_{1-\hat{E}_{t h}+\hat{\epsilon}}^{\infty} \hat{E}_{0}^{-\delta} \int_{1-\hat{E}_{t h}}^{\hat{E}_{0}-\hat{\epsilon}} \frac{\ln \left(\left(\hat{E}_{0}-\hat{V}\right) / \hat{\epsilon}\right)}{\hat{E}_{0}-\hat{V}}\left(\hat{E}_{t h}+\hat{V}\right)^{\delta-1} d \hat{V}^{-\hat{E}} \hat{E}_{0} .
\end{aligned}
$$

Numerical computations of $f\left(\hat{\epsilon}, \delta, \hat{E}_{t h}\right)$ verses $\hat{\epsilon}$ for five values of the electron power-law index $\delta$ are shown in Figure 7. The value of $\hat{E}_{t h}$ is taken to be $0.108(2.15 \mathrm{keV} / 20 \mathrm{keV})$. The value of $f\left(\hat{\epsilon}, \delta, \hat{E}_{t h}\right)$ decreases as $\hat{E}_{t h}$ approaches 1 , since $x_{r c}$ and, therefore, the electron flux density beyond $x_{r c}$ decreases as $E_{t h}$ increases. The increasing values of $f\left(\hat{\epsilon}, \delta, \hat{E}_{t h}\right)$ as $\delta$ increases may be surprising, since the electron flux density beyond $x_{r c}$ decreases as $\delta$ increases (Figure 1). However, the magnitude of the potential drop beyond $x_{r c}$ also decreases with increasing $\delta$ (Figure 2) and, therefore, the electron energy loss rate is smaller. The result is that more photons are emitted per electron and this dominates over the increased loss of electron flux density.

Equation 40 is an upper limit to the integrated X-ray brightness if $n_{i} T^{3 / 2}$ is the highest value of this quantity along the path of the streaming electrons. A lower value of $n_{i}$ means fewer ions on which to scatter and, therefore, lower emission. A lower value of $T$ means a higher resistivity, greater electron energy losses and lower X-ray emission. The inclusion of collisional losses would also decrease the emission. However, this upper limit depends on the values of several physical parameters and, according to the numerical results obtained here, is $\sim 200-500$ times greater than $10^{-15}$ photons $\mathrm{cm}^{-2} \mathrm{~s}^{-1} \mathrm{~cm}^{-2}$. 


\section{Comparison with Numerical Simulations}

The analytical results obtained here are well suited for obtaining a deeper understanding of the numerical results of Zharkova \& Gordovskyy (2006) (hereafter, ZG06). These authors compute steady-state electron distributions and resulting photon spectra for electrons experiencing both return-current and collisional losses. The plasma resistivity is assumed to be classical. Their electron distribution does include a spread in pitch angle, but magnetic mirroring is not included and, as I show in the next two paragraphs, their electron distribution is nearly one-dimensional (1-D).

ZG06 took the pitch-angle dependence of their electron distribution to be proportional to $\exp \left\{-[(\mu-1) / \Delta \mu]^{2}\right\}$ (defined in Zharkova \& Gordovskyy 2005), where $\mu$ is the cosine of the electron pitch angle and $\Delta \mu$ is taken to have the value 0.2 . This electron distribution is smaller by $e^{-25}$ at $90^{\circ}$ than its value at $0^{\circ}$, highly concentrating the electron pitch angles around $0^{\circ}$.

The effectively 1-D character of their electron distribution is further demonstrated by the plots of return-current electric field strength (normalized to the Dreicer electric field) versus column density in their Figure 1. The electric field strength is constant (with a slight rise to be discussed below) up to a column density that decreases with increasing electron energy flux density. It then sharply drops off with increasing column density. This behavior is characteristic of the 1-D model, as seen in Figure 1. The sharp drop-off occurs at the column density $N_{r c}$ corresponding to $x_{r c}$. When electrons are decelerated by the return-current electric field to the point that their pitch angle reaches $90^{\circ}$, they begin to propagate in the $-x$ direction and are lost from the downward-streaming electron beam. This decreases the beam flux density as the electrons are lost, resulting in a corresponding decrease in the return-current electric field strength with distance at distance less than $x_{r c}$ (and column densities less than $N_{r c}$ ) and a smoother drop-off at $x_{r c}$. The absence of this 
decline in the ZG06 electric field plots indicates that the electron flux density distribution can be treated as being 1-D.

\subsection{Return-Current Electric Field Strength}

The injected electron flux density energy distribution used by ZG06 is a single power-law with a low-energy cutoff at $E_{c}=8 \mathrm{keV}$ and a high-energy cutoff at $384 \mathrm{keV}$. Collisional losses will affect these electrons and, therefore, the return-current electric field at column densities $N \gtrsim E_{c}^{2} / 2 K \simeq 1 \times 10^{19} \mathrm{~cm}^{-2}$. In Figure 1(c) of ZG06, for an injected

electron energy flux density of $P_{e 0}=1 \times 10^{12} \mathrm{erg} \mathrm{s}^{-1} \mathrm{~cm}^{-2}, N_{r c} \simeq 1 \times 10^{18} \mathrm{~cm}^{-2}$. Therefore, collisional losses should not be important in this case for column densities on the order of and less than $N_{r c}$.

We have seen here that the electric field strength at distances less than $x_{r c}$ is $E_{r c 0}=\eta e F_{e 0}$ (Equation 8) and is independent of the power-law index of the injected electron flux density distribution. In ZG06's Figure 1, however, $E_{r c} / E_{D}$ below $N_{r c}$ for $\delta=7$ is somewhat greater than for $\delta=3$. (In ZG06, $\gamma$ denotes the electron distribution power-law index and $\delta$ denotes the photon spectral index. Here, as elsewhere in this paper, I write $\delta$ for the electron index and $\gamma$ for the photon index, the more common usage.) This is because the electron energy flux density, rather than the number flux density, is specified in each of the three panels (and throughout the paper). From Equation $27, F_{e 0}(\delta=7) / F_{e 0}(\delta=3)$ for fixed values of $P_{e 0}$ and $E_{c}$ is 1.67. (For $P_{e 0}=1 \times 10^{12} \mathrm{erg} \mathrm{s}^{-1} \mathrm{~cm}^{-2}, E_{c}=8 \mathrm{keV}$, and $\delta=3, F_{e 0}=3.91 \times 10^{19}$ electrons $\mathrm{s}^{-1} \mathrm{~cm}^{-2}$.) This at least qualitatively explains the differences in the initial values of $E_{r c} / E_{D}$. It also follows from Equation 10, however, that $x_{r c}(\delta=3) / x_{r c}(\delta=7)=F_{e 0}(\delta=7) / F_{e 0}(\delta=3)=1.67$. It is interesting that this difference is not apparent in their Figure 1. 
Unfortunately, ZG06 do not explicitly show the plasma temperature and density distributions used in their computations. From their Figure 1(c) we find that $N_{r c} \simeq 1 \times 10^{18} \mathrm{~cm}^{-2}$ and $E_{r c 0} / E_{D} \simeq 150$. (Note that, for this high value of the ratio of $E_{r c 0}$ to $E_{D}$, the plasma resistivity is expected to be enhanced above the classical value and the evolution of the beam/return-current system may not be simple. For this comparison, however, the assumption of classical resistivity is appropriate.) ZG06 define $E_{D}$ as $2 \pi e^{3} n \lambda / k T$ (this is a factor of 2 smaller than the definition in Holman (1985)). Taking the plasma density to be approximately constant in the coronal part of the loop and using Equation 10 for $x_{r c}$, these equations together give $n\left(E_{c}-E_{t h}\right) / e E_{D}=k T\left(E_{c}-E_{t h}\right) /\left(2 \pi e^{4} \lambda\right) \simeq 1.5 \times 10^{20} \mathrm{~cm}^{-2}$. No solution can be obtained with $E_{t h} \sim k T$. Taking $E_{t h}=0$ gives $T \simeq 570 \mathrm{MK}, n \approx 1 \times 10^{7} \mathrm{~cm}^{-3}$, and $x_{r c} \approx 1 \times 10^{11} \mathrm{~cm}$. These are extreme values for an actual flare loop! Also, $570 \mathrm{MK}$ corresponds to $k T \simeq 49 \mathrm{keV}$, which is not consistent with the value of $8 \mathrm{keV}$ for $E_{c}$ when collisional losses in the hot plasma are taken into account. Nevertheless, these are the values I obtain that allow comparison with ZG06's electric field results in Figure 1(c).

A plot of $E_{r c} / E_{D}$ for $\delta=3$ and $\delta=7$ as a function of the column density $N=n x$ for these values of $T$ and $n$ is shown in Figure 8(a). As discussed above, the difference in the values of $N_{r c}=n x_{r c}$ and of $E_{r c 0} / E_{D}$ for $\delta=3$ and $\delta=7$ are greater than in Figure 1(c) of ZG06. Also, for $N>N_{r c}, E_{r c} / E_{D}$ does not drop off nearly as fast with increasing $N$ as in Figure 1(c). Instead, $E_{r c} / E_{D}$ falls off as $N^{-(\delta-1) / \delta}$ (see Section 2.2). The results obtained here and by ZG06 agree qualitatively, however, in that $E_{r c} / E_{D}$ for $\delta=7$ falls off more rapidly than for $\delta=3$.

The differences between the results obtained here and those of ZG06 can be understood in terms of spatial variations in the plasma temperature and density. The slight bump in $E_{r c} / E_{D}$ just before it begins to decrease with $N$ must result from the plasma temperature 
beginning to decrease significantly at this column density, since $E_{r c} / E_{D} \propto F_{e 0} /\left(n T^{1 / 2}\right)$. Since $n$ increases with column density, only a decrease in $T$ can cause an increase in $E_{r c} / E_{D}$. The more rapid decrease of $E_{r c} / E_{D}$ with $N$ in Figure $1(\mathrm{c})$ must be caused be an increase in plasma density with $N$. Collisional losses will also cause a more rapid decrease, but, as discussed above, this should not be significant at $N \approx 10^{18} \mathrm{~cm}^{-2}$.

Figure $8(\mathrm{~b})$ shows an example of the effect of spatially varying plasma temperature and density on the dependence of $E_{r c} / E_{D}$ on $N$. The plasma temperature is taken to decrease exponentially as $T_{0} \exp \left(-x / x_{T}\right)$, with $T_{0}=570 \mathrm{MK}$ and $x_{T}=30 \mathrm{Mm}$. The density increases exponentially as $n_{0} \exp \left(x / x_{n}\right)$, with $n_{0}=9 \times 10^{7} \mathrm{~cm}^{-3}$ and $x_{n}=100 \mathrm{Mm}$. As before, $E_{c}=8 \mathrm{keV}, E_{t h}=0$, and $P_{e 0}=1 \times 10^{12} \mathrm{erg} \mathrm{s}^{-1} \mathrm{~cm}^{-2}$. The column density is now $N(x)=n_{0} L_{n}\left(e^{x / L_{n}}-1\right)$. From Equation 19 and the definition of $\rho\left(x_{r c}\right)$, $x_{r c}=\frac{2}{3} L_{T} \ln \left(1+\frac{3}{2} \frac{x_{r c 0}}{L_{T}}\right)$, where $x_{r c 0}$ is the value of $x_{r c}$ for $T=T_{0}$ and for the value of $F_{e 0}$ corresponding to the value of $\delta$. The column density corresponding to $x_{r c}$ is now given by $N_{r c}=n_{0} x_{n}\left[\left(1+\frac{3}{2} \frac{x_{r c 0}}{x_{T}}\right)^{\frac{2 x_{T}}{3 x_{n}}}-1\right]$. Vertical lines show the values of $N_{r c}$ for $\delta=3$ and $\delta=7$, as in Figure 8(a).

There is now an increase in $E_{r c} / E_{D}$ below $N_{r c}$, similar to the increase in ZG06's Figure 1. This is caused by the decrease in temperature with distance. This same decrease in temperature decreases, rather than increases, the rate of decline of $E_{r c} / E_{D}$ beyond $N_{r c}$. A substantially more rapid rate of decline is obtained because of the increase in density with distance, however. This rapid decline is entirely due to the increase in $E_{D}$ with distance. The difference between $N_{r c}(\delta=3)$ and $N_{r c}(\delta=7)$ is now substantially smaller than in Figure 8(a), more consistent with the results of ZG06.

Figure 1(c) of ZG06 shows a flattening of $E_{r c} / E_{D}$ at higher values of $N$, most notably for $\delta=3$ in the range $N \approx 10^{20}-10^{22} \mathrm{~cm}^{-2}$. This could be associated with a more rapid decline in the plasma temperature and/or a less rapid increase in the plasma density. A 
decrease in the plasma ionization could also contribute to the flattening.

\subsection{X-Ray Brightness Spectra}

ZG06 show computed photon spectra in their Figure 10, and results derived from these spectra in Figures 11 and 12. Here I compute thick-target bremsstrahlung X-ray brightness spectra from the 1-D return-current electron flux density distribution function, Equation 12, and compare the computed spectra with the results of ZG06. As discussed in Section 2.6, I assume that return-current losses are solely important up to the thick-target region at distance $x_{t t}$, and insignificant beyond $x_{t t}$, where collisional losses dominate. As in Section 3.1, these assumptions are most likely to be valid for the highest electron energy flux density considered by ZG06, $10^{12} \mathrm{erg} \mathrm{s}^{-1} \mathrm{~cm}^{-2}$.

With these assumptions and use of the Kramers bremsstrahlung cross section, it is possible to obtain relatively simple analytical expressions for comparison with the numerical results. Since the electron distribution of ZG06 contains a high-energy cutoff, denoted here as in ZG06 as $E_{\text {upp }}$, and photon energies below the low-energy cutoff at $E_{c}=8 \mathrm{keV}$ are considered, I extend Equation 37 to account for these cutoffs:

$$
\begin{gathered}
B(\epsilon)=1.17 \times 10^{-34} F_{e 0}\left(\frac{\delta-1}{\epsilon}\right) \times \\
\begin{cases}{\left[\frac{E_{c}}{\delta-2}-\frac{\epsilon+V_{t t}}{\delta-1}-\left(\frac{E_{c}}{E_{u p p}}\right)^{\delta-1}\left(\frac{E_{u p p}}{\delta-2}-\frac{\epsilon+V_{t t}}{\delta-1}\right)\right]} & \epsilon<\max \left[E_{t h}, E_{c}-V_{t t}\right] \\
{\left[\frac{E_{c}^{\delta-1}\left(\epsilon+V_{t t}\right)^{2-\delta}}{(\delta-1)(\delta-2)}-\left(\frac{E_{c}}{E_{u p p}}\right)^{\delta-1}\left(\frac{E_{u p p}}{\delta-2}-\frac{\epsilon+V_{t t}}{\delta-1}\right)\right]} & \max \left[E_{t h}, E_{c}-V_{t t}\right] \leq \epsilon<E_{u p p}-V_{t t} \\
0 & \epsilon \geq E_{u p p}-V_{t t},\end{cases}
\end{gathered}
$$

where $V_{t t}$ is shorthand for $V\left(x_{t t}\right)$.

The spectra in Figure 10 of ZG06 are normalized to their value at $4 \mathrm{keV}$ and are computed for $\delta=3$ and $\delta=7$. The only free parameter in the 1-D model for comparison 
to these results is the value of the potential drop at $x_{t t}, V_{t t}$, since the low- and high-energy cutoffs of the electron flux distribution injected at $x=0$ are fixed at $E_{c}=8 \mathrm{keV}$ and $E_{\text {upp }}=384 \mathrm{keV}$.

In Figure 9 brightness spectra, normalized to their value at $4 \mathrm{keV}$, are computed using Equation 42 and plotted for several values of $V_{t t}$ for both values of $\delta$. Points (diamond symbols) from Figure 10(b) of ZG06, for $P_{e 0}=10^{12} \mathrm{erg} \mathrm{s}^{-1} \mathrm{~cm}^{-2}$, are shown for comparison. None of the curves provide a good fit to the ZG06 results. For $\delta=3$, both $V_{t t}=15 \mathrm{kV}$ and $V_{t t}=315 \mathrm{kV}$ provide a reasonable fit up to about $40 \mathrm{keV}$. For $\delta=7, V_{t t}=12 \mathrm{kV}$ provides a reasonable fit up to about $60 \mathrm{keV}$. The Kramers cross section is not best suited for computing spectra from electron distributions containing cutoffs, however (cf. Brown et al. 2008).

For a more accurate comparison I have computed the X-ray spectra using the thick-target fitting function in the RHESSI data analysis software, which uses the Haug (1997) bremsstrahlung cross section (cf. Holman 2003; Holman et al. 2003). The default double-power-law electron distribution function is replaced with Equation 12, with the addition of a sharp high-energy cutoff at $E_{u p p}-V_{t t}$ and with $V_{t t}$ replacing $V(x)$. The results are shown in Figure 10. Better fits to the ZG06 results are obtained, but only at energies of $100 \mathrm{keV}$ and below. Agreement is not good above $100 \mathrm{keV}$, especially for $\delta=3$. These fits give $V_{t t} \simeq 130 \mathrm{kV}$ for $\delta=3$ and $V_{t t} \simeq 14 \mathrm{kV}$ for $\delta=7$. The discrepancy is particularly evident for $\delta=3$. For $V_{t t}=130 \mathrm{kV}$, the high-energy cutoff is at $384-130=254 \mathrm{keV}$. The ZG06 spectrum shows emission well above $254 \mathrm{keV}$, however.

It is interesting to compare these deduced values for $V_{t t}$ with values expected from the simple constant density and temperature model obtained in Section 3.1, represented by the electric field plot in Figure 8(a). The corresponding potential drop curves for $\delta=3$ and 7 are plotted in Figure 11. The dotted diagonal line shows the column density $N=\frac{E^{2}}{2 K}$ 
(Equation 30) at which an electron of energy $E=V$ looses all its energy from collisional losses alone. Since the electrons injected at $N=0$ range in energy from 8 to $384 \mathrm{keV}$ (horizontal dotted lines), the thick target region (without return-current losses) ranges from about $1.1 \times 10^{19} \mathrm{~cm}^{-2}$ to $2.5 \times 10^{22} \mathrm{~cm}^{-2}$ (vertical dotted lines). The intersection of the potential drop curves with the start of the thick-target region gives $V_{t t} \simeq 22 \mathrm{kV}$ for $\delta=3$ and $V_{t t} \simeq 15 \mathrm{kV}$ for $\delta=7$.

The simple model is, of course, much too simple for quantitative conclusions. The result for $V_{t t}$ when $\delta=7$ agrees rather well with the result from the spectral comparison, however. The results also agree qualitatively in that $V_{t t}$ for $\delta=3$ is larger than $V_{t t}$ for $\delta=7$ in both cases. But the quantitative results for $\delta=3$ do not agree. The model assumption that $V(N)$ is constant in the thick-target region is clearly violated for $\delta=3$. The increase of $V$ with $N$ will be diminished by collisional losses and by the significant decrease in the value of $E_{\text {upp }}-V(N)$. Nevertheless, this flat electron distribution is more likely to violate the model assumptions than the steep distribution with $\delta=7$, since in the thick-target region $V(N)$ is increasing as $N^{1 / \delta}$ for return-current losses alone (for $n$ constant and $N \gg N_{r c}$; see the discussion after Equation 16). Further comparisons with numerical models that combine return-current and collisional losses are needed to test the realm of validity of this simple model.

\subsection{X-Ray Spectral Index}

The thick-target X-ray spectrum with return-current losses taken into account, approximated by Equation 42, contains substantial deviations from the single-power-law photon spectrum, $B(\epsilon)=B_{0}\left(\epsilon / \epsilon_{0}\right)^{-\gamma}$. These deviations are caused by the return-current energy losses, but they are also caused by the presence of a low-energy cutoff $\left(E_{c}\right)$ and a high-energy cutoff $\left(E_{\text {upp }}\right)$ in the injected electron distribution. The local spectral index at 
energy $\epsilon, \gamma(\epsilon)=-d \log B(\epsilon) / d \log \epsilon=-(\epsilon / B)(d B / d \epsilon)$, can be obtained analytically from Equation 42. The result is

$$
\begin{aligned}
& \gamma(\epsilon)=1+\left(\frac{\epsilon}{\delta-1}\right) \frac{1-\left(\frac{E_{c}}{E_{u p p}}\right)^{\delta-1}}{\frac{E_{c}}{\delta-2}-\frac{\epsilon+V_{t t}}{\delta-1}+\left(\frac{E_{c}}{E_{u p p}}\right)^{\delta-1}\left(\frac{\epsilon+V_{t t}}{\delta-1}-\frac{E_{u p p}}{\delta-2}\right)} \\
& \gamma(\epsilon)=1+\epsilon \frac{\left(\epsilon+V_{t t}\right)^{1-\delta}-E_{u p p}^{1-\delta}}{\frac{\left(\epsilon+V_{t t}\right)^{2-\delta}}{\delta-2}+E_{u p p}^{1-\delta}\left(\epsilon+V_{t t}-\frac{\delta-1}{\delta-2} E_{u p p}\right)} \quad \max \left[E_{t h}, E_{c}-V_{t t}\right] \leq \epsilon<E_{u p p}-V_{t t} .
\end{aligned}
$$

For no high-energy cutoff $\left(E_{u p p} \rightarrow \infty\right)$, these equations reduce to

$$
\begin{aligned}
& \gamma(\epsilon)=1+(\delta-2) \frac{\epsilon}{(\delta-1) E_{c}-(\delta-2)\left(\epsilon+V_{t t}\right)} \quad \epsilon<\max \left[E_{t h}, E_{c}-V_{t t}\right] \\
& \gamma(\epsilon)=1+(\delta-2) \frac{\epsilon}{\epsilon+V_{t t}} \quad \max \left[E_{t h}, E_{c}-V_{t t}\right] \leq \epsilon<E_{u p p}-V_{t t} .
\end{aligned}
$$

Plots of $\gamma(\epsilon)$ computed from Equations 43 and 44 are shown in Figure 12 for $\delta=3$ (top panel) and $\delta=7$ (bottom panel). The top dashed curve in each panel shows $\gamma(\epsilon)$ for no high-energy cutoff and $V_{t t}=0 \mathrm{kV}$. The low-energy cutoff for all curves is $E_{c}=8 \mathrm{keV}$. Above $\epsilon=E_{c}$ the spectrum has the expected thick-target power-law dependence with $\gamma=\delta-1$. The dotted curves show $\gamma(\epsilon)$ with $E_{\text {upp }}=384 \mathrm{keV}$. For $\delta=7$ the power-law dependence of the spectrum is retained below $\sim 100 \mathrm{keV}$. For $\delta=3$, no substantial part of the spectrum is well described by a single power law. For an electron distribution this flat, the X-ray emission from electrons with energies as high as $384 \mathrm{keV}$ is still significant at photon energies as low as $8 \mathrm{keV}$.

The three solid curves in each panel of Figure 12 are for $V_{t t}=14,130$, and $260 \mathrm{kV}$, respectively. The three remaining dashed curves show the corresponding result when there is no high-energy cutoff. Although $14 \mathrm{kV}$ is a relatively small potential drop, it significantly affects most of the X-ray spectrum over the plotted range of photon energies. For the solid curves, the high-energy cutoff at $x_{t t}$ has been reduced from $384 \mathrm{keV}$ to 370,254 , and 
$124 \mathrm{keV}$, respectively. Comparison of the solid and dashed curves shows the substantial impact of this high-energy cutoff on the X-ray spectrum, especially for $\delta=3$.

\section{Spectral Evolution and the Return-Current "Bump"}

ZG06 show plots of the photon spectral index at 20 and $100 \mathrm{keV}$ and their difference versus three values of the electron flux density distribution power-law index and three values of the injected electron energy flux density. Since the power-law index and the injected energy flux density are not observable quantities, here I will look at the dependence of $V_{t t}$ and spectral index, $\gamma$, on the hard X-ray brightness, $B(\epsilon)$, quantities that can be deduced from spectral fits. These quantities are computed as a function of the injected electron flux density, $F_{e 0}$, assuming that all changes are due to changes in $F_{e 0} . F_{e 0}$ increases from $10^{15}$ to $10^{21}$ electrons $\mathrm{s}^{-1} \mathrm{~cm}^{-2}$. The plasma temperature and the distance to the top of the thick-target region, $x_{t t}$, are taken to be $10 \mathrm{MK}$ and $3 \times 10^{9} \mathrm{~cm}$, respectively. The low-energy cutoff is $20 \mathrm{keV}$ and the high-energy cutoff is taken to be high enough to not affect the results (i.e., no high-energy cutoff).

Figure 13 shows how $V_{t t}$ varies with $B(\epsilon)$ for three values of the injected electron flux density distribution power law index and for the X-ray brightness measured at two different photon energies. Where $V_{t t} \ll E_{c}-E_{t h}=17.85 \mathrm{keV}, V_{t t}$ and, therefore, the effective low-energy cutoff, increases (or decreases) linearly with $B(\epsilon)$. In this regime both $V_{t t}$ and $B(\epsilon)$ are directly proportional to $F_{e 0}$. At higher photon energies and values of $\delta$, the X-ray brightness is lower for a given value of $V_{t t}$ (and $F_{e 0}$ ). This linear relationship is lost when $V_{t t} \simeq E_{c}-E_{t h}$. When $V_{t t} \gg E_{c}-E_{t h}, V_{t t}$ increases more slowly with $B(\epsilon)$ or, conversely, $B(\epsilon)$ increases more rapidly with increasing $V_{t t}$.

An interesting feature appears where $V_{t t} \simeq E_{c}-E_{t h}$ in the curves for $\epsilon=20 \mathrm{keV}$ and 
$\delta=5$ and 7. For this lower value of $\epsilon$ and higher values of $\delta$, as $V_{t t}$ increases $B(\epsilon)$ decreases somewhat and reaches a local minimum value before continuing to increase at a faster rate. This behavior is similar to that in Figure 6. This would be observed as a small sub-peak, or "bump", on the rise (or fall) of the impulsive peak of a flare, followed by a more rapid increase in the X-ray brightness with increasing $V_{t t}$. The increase in the rate of brightening would be seen at all photon energies, but the sub-peak would only appear at lower energies. This may provide a signature of return-current losses in flare light curves.

Figure 14 shows the evolution of spectral index with X-ray brightness for the same parameters as in Figure 13. As $F_{e 0}$ and $B(\epsilon)$ increase, $\gamma$ remains constant until $V_{t t}$ becomes high enough to significantly decrease the energy of electrons of energy $\epsilon$ and above $\left(V_{t t} \gtrsim 0.1 \epsilon\right)$. The spectrum then flattens with increasing rapidity until $V_{t t}=E_{c}-E_{t h}$, after which the spectrum continues to flatten, but at a lower rate. Only the spectral index at $20 \mathrm{keV}$ for $\delta=3$ comes close to the lowest possible value, $\gamma=1$. The dip in brightness seen in Figure 13 for $\delta=5$ and 7 is also seen here as the spectrum flattens.

To explore more directly the possibility of seeing this bump in X-ray light curves, I end this section by looking at the dependence of $B(\epsilon)$ on $F_{e 0}$. This dependence is representative of the X-ray light curve if $F_{e 0}$ varies linearly with time or, at least, not too differently from a linear dependence. Figure 15 shows this dependence for $\delta=5$ (top panel) and $\delta=7$ (bottom panel). Each panel shows curves for nine values of the photon energy, starting at $\epsilon=20 \mathrm{keV}$ (top curve) and increasing in steps of $10 \mathrm{keV}$ to $\epsilon=100 \mathrm{keV}$ (bottom curve). The brightness increases linearly with $F_{e 0}$ for $V_{t t}<E_{c}-E_{t h}$, and as $F_{e 0}^{2 / \delta}$ for $V_{t t} \gg E_{c}-E_{t h}$. In addition to this flattening of the dependence, the return-current bump appears in the curves at photon energies $\epsilon$ less than $(\delta-3)\left(E_{c}-E_{t h}\right)$. It is interesting that there is no sub-peak when $\delta \leq 3$. When $\epsilon$ satisfies this condition, the value of $F_{e 0}$ at the local maximum is on the order of and somewhat smaller than $F_{e 0}^{t t}$ (Equation 33). These interesting features may provide a 
valuable tool for recognizing return-current losses in flare data.

\section{Summary and Conclusions}

The 1-D, steady-state model examined here demonstrates how return-current losses can have a substantial impact on the observed X-ray emission from flares, primarily through the flattening of the nonthermal electron distribution function at low energies and enhanced heating in the coronal part of flare loops. Both the electron energy losses and the heating rate are sensitive to the injected electron flux density, $F_{e 0}$. Since $F_{e 0}$ is sensitive to the low-energy cutoff, $E_{c}$, to the injected electron flux density energy distribution, the results are also sensitive to the value of $E_{c}$.

When the energy of the nonthermal electrons becomes low enough because of deceleration by the return-current electric field and collisional losses, they are thermalized and lost from the beam. When enough electrons are thermalized to significantly decrease the electron beam flux density, return-current losses and the heating rate begin to decrease with distance from the injection point. The distance at which this occurs, $x_{r c}$ (Equation 10), is sensitive to $E_{c}$ and $F_{e 0}$ and to the approximate energy at which the electrons are thermalized, $E_{t h}$, if it is comparable to $E_{c}$. The plasma resistivity is also important, which, classically, is determined primarily by the plasma temperature. This evolution is only sensitive to the plasma density, however, where collisional losses are important. If $x_{r c}$ becomes smaller than the distance to the thick-target footpoints where most of the hard $\mathrm{X}$-ray emission is emitted, this decrease in the rate of return-current losses has interesting observational consequences.

The flattening of the electron distribution by return-current losses results in a new effective low-energy cutoff to the electron distribution that increases with distance $x$ from 
the injection point. This low-energy cutoff is the potential drop $V(x)$ corresponding the the integral of the return-current electric field from the injection point to $x$. $V$ increases linearly with the column resistivity $\rho$ (Equation 17) until the distance $x_{r c}$ is reached or, more generally, until the corresponding column resistivity $\rho_{r c}$ is reached (Equation 19). Beyond this distance $V$ increases at a much lower rate. This rate is further reduced by collisional losses (Section 2.5).

How do we best recognize return-current losses in solar flare data, and how do we use this recognition to deduce the electron flux density distribution injected from the acceleration region, the subsequent evolution of the accelerated electrons, and their impact on the flare plasma? As I briefly review in the Introduction, several methods and possible identifications of return-current losses are already presented in the literature.

Zharkova \& Gordovskyy (2006) emphasized comparing the spectral index at a low and a high photon energy with numerical model results. Such a comparison was done for two spectra from two flares in Zharkova et al. (2010). For each case an estimate of the electron energy flux density at the times of the two spectra was obtained. This approach, however, does not take full advantage of the spectral shape and does not provide a test of the plausibility of the model. The spectral evolution for one of the flares, 2002 July 23 for example, has been found by Kontar et al. (2003) to be consistent with nonuniform ionization of the target plasma. The spectral indices, as shown in Section 3.3, are also sensitive to assumptions about low- and high-energy cutoffs to the injected electron flux density distribution.

Alexander \& Daou (2007) found evidence for the upper limit of $10^{-15}$ photons $\mathrm{cm}^{-2} \mathrm{~s}^{-1}$ $\mathrm{cm}^{-2}$ to the integrated X-ray brightness above $20 \mathrm{keV}$, deduced by Emslie (1980) to result from return-current losses. In Section 2.6 I find that this is not an upper limit but, rather, is roughly the point at which the rate of increase of brightness with increasing injected 
electron flux density significantly decreases because of the thermalization of beam electrons. Nevertheless, return-current losses should be important when the integrated brightness is of this magnitude and, therefore, this can be a valuable indicator that return-current losses are significant. A difficulty with this test is that X-ray images can only provide an upper limit to the area of the emission and, therefore, a lower limit to the integrated X-ray brightness. Also, it is not clear why Alexander \& Daou (2007) did not find any integrated source brightnesses greater than this estimated value.

Comparison of coronal and footpoint hard X-ray spectra, as in Battaglia \& Benz (2008), provides an excellent tool for identifying return-current losses. As pointed out by Hannah \& Kontar (2011), however, another loss mechanism such as the bump-on-tail instability could be responsible for energy loss between the coronal source and the footpoints. The scarcity of observed nonthermal coronal X-ray sources also limits the usefulness of this method.

An important characteristic of return-current losses is the flattening of the X-ray spectrum at low photon energies resulting from the flattening of the electron distribution at low energies. This flattening has the character of a low-energy cutoff. The X-ray spectrum flattens more slowly with decreasing photon energy than for a sharp low-energy cutoff. This difference would be difficult to detect with current observations, however. Also, a similar spectral flattening could result from a plasma instability such as the bump-on-tail instability.

The most distinctive characteristic of return-current losses is the spectral evolution. The spectrum flattens to higher energies with both distance from the injection point and with increasing injected electron flux density, $F_{e 0}$. Flattening of the spectrum with distance is also a characteristic of collisional losses, but flattening of the integrated spectrum with increasing $F_{e 0}$ is not a characteristic of collisional losses. Thus, as the X-ray brightness increases, so does the effective low-energy cutoff. This behavior has been 
noted in a sample of flare spectra studied by Sui et al. (2007). Although this evolution is distinctive, it might also be produced in the acceleration region or, possibly, by a plasma instability. Therefore, appropriate spectral evolution, together with one or more additional observational characteristics, is most appropriate for confidently identifying return-current losses.

The return-current "bump" and reduced rate of rise (increased rate of fall) in hard X-ray light curves as peak brightness is approached (as the brightness declines) is another feature that can be used to identify return-current losses (Section 4 and Figure 15). Related features could also be identified in the time evolution of the low-energy cutoff (Figure 13) and X-ray spectral index (Figure 14). A limitation of these identifiers is that this evolution is a function of $F_{e 0}$, a quantity not directly obtained from observations and, therefore, with a time evolution that is not directly known. On the other hand, identification of the bump and its dependence on photon energy in light curves could be a strong indicator of return-current losses.

Perhaps the most interesting physical characteristic of return-current losses is that spectral fitting to deduce the value of the low-energy cutoff provides a direct measure of the potential drop in the flare loop. This is best done (in the context of the 1-D, analytical model at least) by fitting the computed bremsstrahlung emission from Equation 12 to the hard X-ray spectra. The time evolution of $V_{t t}$ determined from these spectral fits gives the time evolution of the potential drop. If the low-energy cutoff to the injected electron flux distribution, $E_{c}$, is not hidden beneath the flare thermal bremsstrahlung emission, so the early spectral evolution can be observed, the derived low-energy cutoff will first decrease as $E_{c}-V_{t t}$ while $V_{t t}$ increases with time as $F_{e 0}$ increases. When $V_{t t}$ becomes comparable to the observed photon energies, the increase in $V_{t t}$ with $F_{e 0}$ should be observed. Should a high-energy cutoff, $E_{\text {upp }}$, be present in the injected electron distribution, it will also decrease 
as $E_{u p p}-V_{t t}$ (Equation 42).

As shown in Equation 18, the initial linear rise of $V_{t t}$ with $F_{e 0}$ is proportional to the column resistivity, $\rho$. Therefore, the time evolution of the potential drop also give the time evolution of the column resistivity. Since the classical resistivity depends primarily on the plasma temperature, this can be compared with observations of the thermal plasma in the flare loop. Of particular interest, an exceptionally high value of the resistivity as compared to the classical resistivity would indicate the presence of anomalous resistivity in the loop.

Unless the early evolution of the spectra at low photon energies is observable, one of the most difficult parameters to deduce is $E_{c}$. As seen in Equation 37, a spectral fit gives $E_{c}^{\delta-1} F_{e 0}$, not $F_{e 0}$ or $E_{c}$ independently. The difference between $E_{c}$ and the thermalization energy, $E_{c}-E_{t h}$, can be estimated, however, if the value of $V_{t t}$ can be determined when its dependence on X-ray brightness begins to change (Section 4). This gives an estimate for $E_{c}$ if $E_{t h} \ll E_{c}$ or if an independent estimate of $E_{t h}$ can be obtained.

An additional test for return-current losses is to compare the results deduced from the hard X-ray emission with the thermal evolution of the flare plasma. Direct heating of the coronal plasma by the return current and thermalized electrons can be quite substantial (Section 2.4). For the beam and plasma parameters used in Figure 3, the heating rate in a coronal volume of $10^{25} \mathrm{~cm}^{3}$ is $10^{27} \mathrm{erg} \mathrm{s}^{-1}$. As discussed at the end of Section 2.4, the return-current heating rate at distances less than $x_{r c}$ is $67 \%$ of the power carried by the beam (for $E_{t h}=2.15 \mathrm{keV}$ ). Therefore, for relatively high electron beam flux densities, heating to $>10 \mathrm{MK}$ flare temperatures should be more rapid than when return-current losses are not significant. The inclusion of return-current losses in chromospheric evaporation models should reveal more detailed aspects of how the thermal response is modified.

A weakness of the analytical model presented here is that, if the increase in the potential drop within the thick-target region has a significant impact on the resulting X-ray 
emission, the use of the value of the potential drop at the top of the thick-target region, $V_{t t}$, is not valid. The model results must then be computed with collisional and return-current losses contributing simultaneously (as in Emslie 1980). The impact of collisional losses on the model is discussed in Section 2.5. In future work a numerical model including both loss mechanisms together will be developed, and the results compared to those from the analytical model.

A potentially important signature of return-current losses is electrons temporarily trapped in the top of the flare loops by the return-current electric field. In the 1-D model considered here, no electrons are turned around by the electric field, so that they move upward in the loop and are re-accelerated by the electric field. All electrons are thermalized before they can be reflected. Electrons with high enough pitch angles would be reflected and trapped in the loop as long as the electric field is sustained. The acceleration of back-scattered electrons by the return-current electric field, including the presence of magnetic mirroring, has been simulated and discussed by Karlicky (1993) and Karlicky \& Henoux (1993). These are also included in the simulations of Zharkova et al. (2010) and Kuznetsov \& Zharkova (2010). This interesting topic will be addressed in a future paper.

This work was supported by NASA Heliophysics Guest Investigator Grant 09-HGI090064 and by the RHESSI Program. I thank the referee and Miriem Alaoui Abdallaoui for valuable comments and questions that led to improvements in the text. 


\section{REFERENCES}

Alexander, D., \& Daou, A. G. 2007, ApJ, 666, 1268

Aschwanden, M. J., Kosugi, T., Hudson, H. S., Wills, M. J., \& Schwartz, R. A. 1996, ApJ, 470,1198

Battaglia, M., \& Benz, A. O. 2008, A\&A, 487, 337

Brown, J. C. 1971, Sol. Phys., 18, 489

Brown, J. C., \& McClymont, A. N. 1987, Ap\&SS, 133, 297

Brown, J. C., Kašparová, J., Massone, A. M., \& Piana, M. 2008, A\&A, 486, 1023

Dennis, B. R., \& Pernak, R. L. 2009, ApJ, 698, 2131

D'Iakonov, S. V., \& Somov, B. V. 1988, Sol. Phys., 116, 119

Emslie, A. G. 1980, ApJ, 235, 1055

Emslie, A. G. 1981, ApJ, 249, 817

Emslie, A. G. 2003, ApJ, 595, L119

Feldman, U., Hiei, E., Phillips, K. J. H., Brown, C. M., \& Lang, J. 1994, ApJ, 421, 843

Fisher, G. H., Canfield, R. C., \& McClymont, A. N. 1985, ApJ, 289, 414

Hannah, I. G., \& Kontar, E. P. 2011, A\&A, 529, A109

Haug, E. 1997, A\&A, 326, 417

Holman, G. D. 1985, ApJ, 293, 584

Holman, G. D. 2003, ApJ, 586, 606 
Holman, G. D., Sui, L., Schwartz, R. A., \& Emslie, A. G. 2003, ApJ, 595, L97

Holman, G. D., Aschwanden, M. J., Aurass, H., Battaglia, M., Grigis, P. C., Kontar, E. P., Liu, W., Saint-Hilaire, P., Zharkova, V. V. 2011, Space Sci. Rev., 159, 107, DOI: $10.1007 / \mathrm{s} 11214-010-9680-9$

Hoyng, P., van Beek, H. F., \& Brown, J. C. 1976, Sol. Phys., 48, 197

Huba, J. D. 2009, NRL Plasma Formulary

Hurford, G. J., Schwartz, R. A., Krucker, S., Lin, R. P., Smith, D. M., \& Vilmer, N. 2003, ApJ, 595, L77

Hurford, G. J., Krucker, S., Lin, R. P., Schwartz, R. A., Share, G. H., \& Smith, D. M. 2006, ApJ, 644, L93

Karlicky, M., Alexander, D., Brown, J. C., \& MacKinnon, A. L. 1990, Sol. Phys., 129, 325

Karlicky, M. 1993, Sol. Phys., 145, 137

Karlicky, M., \& Henoux, J.-C. 1993, A\&A, 278, 627

Karlický, M., Kašparová, J., \& Heinzel, P. 2004, A\&A, 416, L13

Karlický, M., Nickeler, D. H., \& Bárta, M. 2008, A\&A, 486, 325

Karlický, M. 2009, ApJ, 690, 189

Karlický, M., \& Kašparová, J. 2009, A\&A, 506, 1437

Knight, J. W., \& Sturrock, P. A. 1977, ApJ, 218, 306

Kontar, E. P., Brown, J. C., Emslie, A. G., et al. 2003, ApJ, 595, L123

Kuznetsov, A. A., \& Zharkova, V. V. 2010, ApJ, 722, 1577 
Lee, K. W., Büchner, J., \& Elkina, N. 2008, A\&A, 478, 889

Litvinenko, I. E., \& Somov, B. V. 1991, Sol. Phys., 131, 319

Miller, J. A., et al. 1997, J. Geophys. Res., 102, 14631

Phillips, K. J. H., Bhatia, A. K., Mason, H. E., \& Zarro, D. M. 1996, ApJ, 466, 549

Rowland, H. L., \& Vlahos, L. 1985, A\&A, 142, 219

Siversky, T. V., \& Zharkova, V. V. 2009, A\&A, 504, 1057

Sui, L., Holman, G. D., \& Dennis, B. R. 2007, ApJ, 670, 862

van den Oord, G. H. J. 1990, A\&A, 234, 496

Warmuth, A., Holman, G. D., Dennis, B. R., Mann, G., Aurass, H., \& Milligan, R. O. 2009, ApJ, 699, 917

Zharkova, V. V., \& Gordovskyy, M. 2005, A\&A, 432, 1033

Zharkova, V. V., \& Gordovskyy, M. 2006, ApJ, 651, 553

Zharkova, V. V., Kuznetsov, A. A., \& Siversky, T. V. 2010, A\&A, 512, A8

Zharkova, V. V., \& Siversky, T. V. 2011, ApJ, 733, 33 


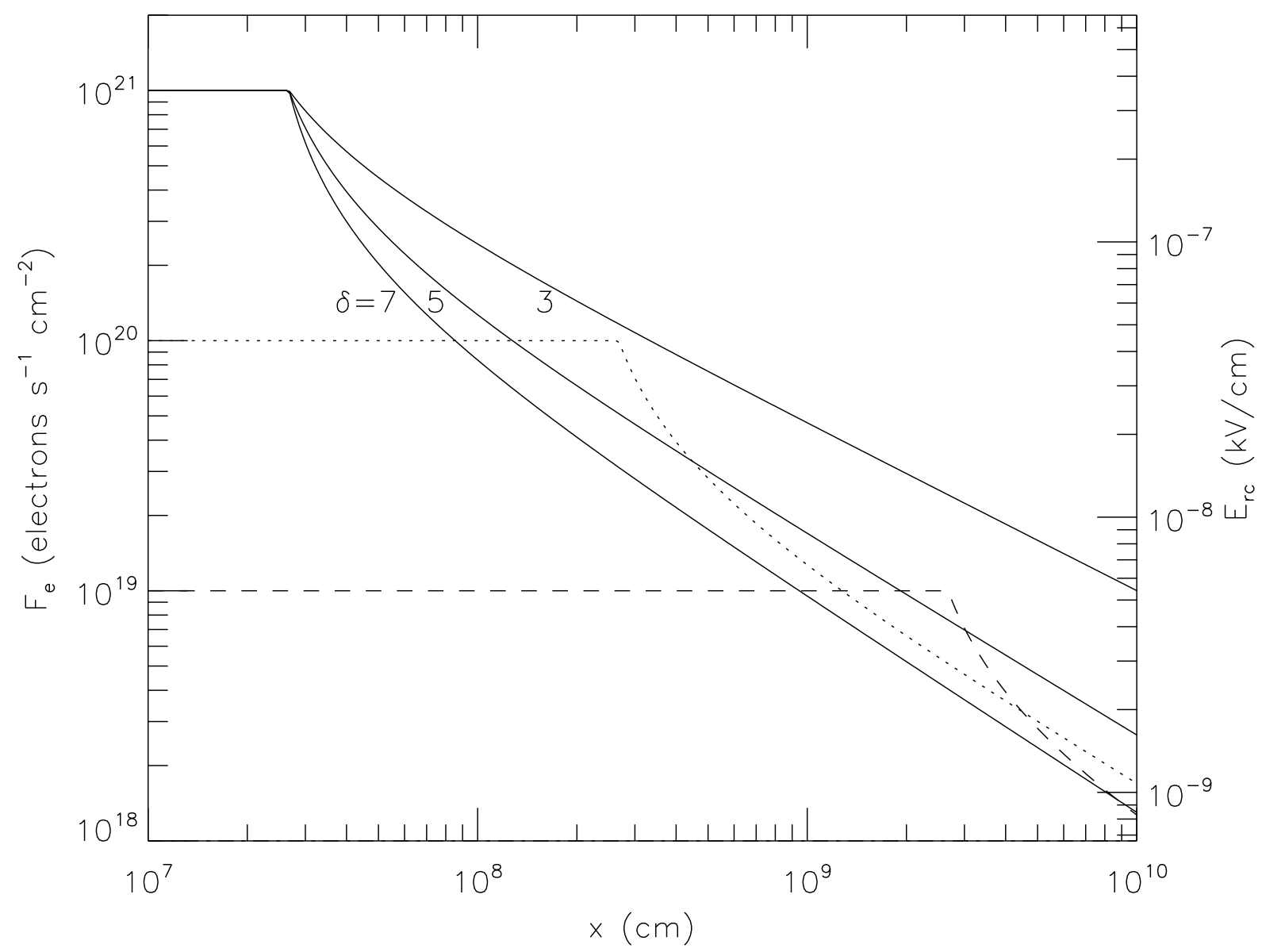

Fig. 1.- Electron flux density (left axis) and return-current electric field strength in kilovolts per centimeter (right axis) are plotted as a function of distance for three values of the injected electron flux density. The results are for $\delta=5$ except for $F_{e 0}=10^{21}$ electrons s${ }^{-1} \mathrm{~cm}^{-2}$, where results for $\delta=3$ and $\delta=7$ are also shown. The plasma temperature is assumed to be constant at $T=10 \mathrm{MK}$ and the default value of $\lambda=20$ is used. The initial cutoff energy is $E_{c}=20 \mathrm{keV}$ and $E_{t h}=2.5 k T=2.15 \mathrm{keV}$. 


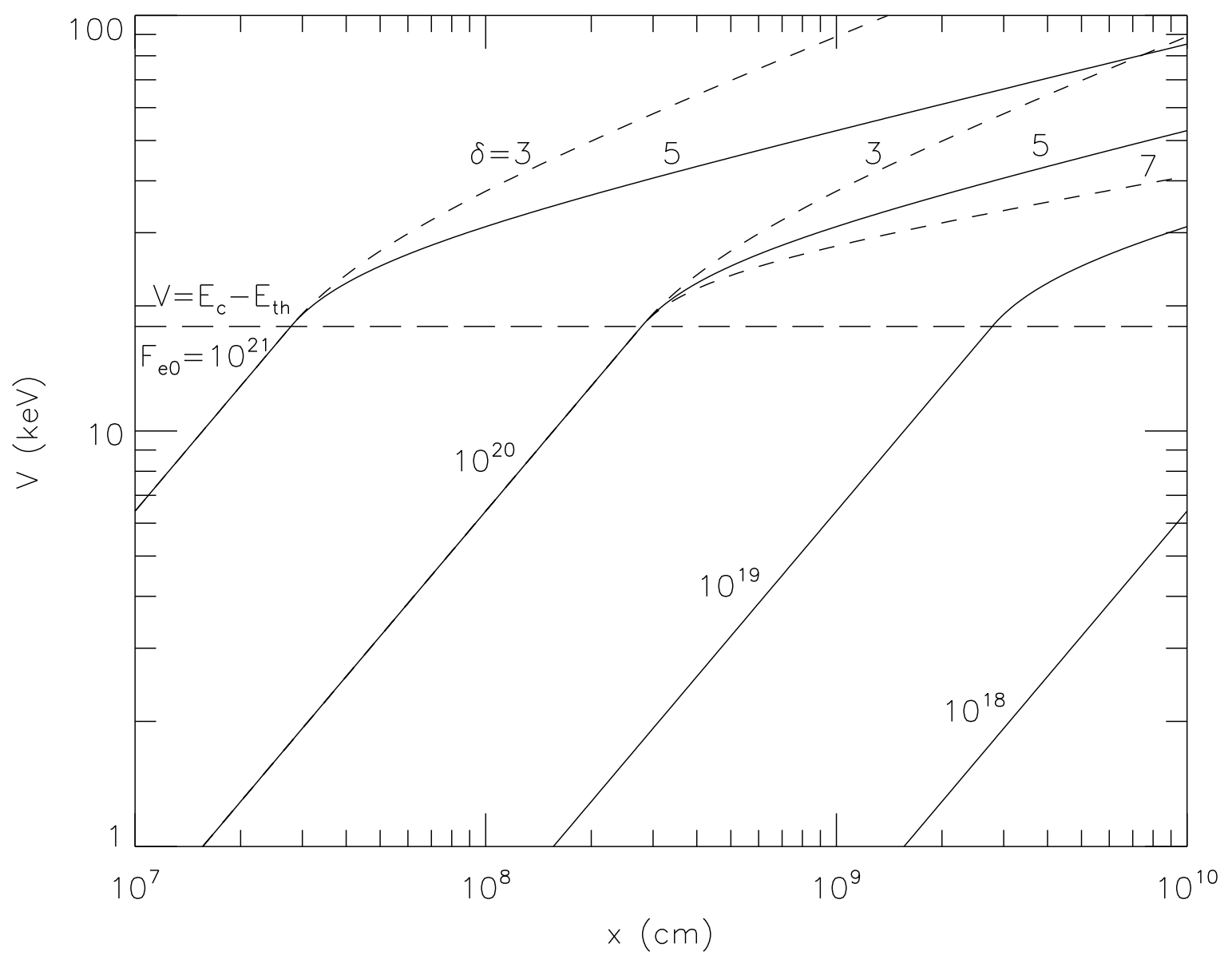

Fig. 2.- Potential drop is plotted as a function of distance for four values of the injected electron flux density. The solid curves are for $\delta=5$. Several curves for $\delta=3$ and $\delta=7$ are also shown (short dashes). Other parameters are the same as in Figure 1. The horizontal line (long dashes) shows the value of $E_{c}-E_{t h}$. 


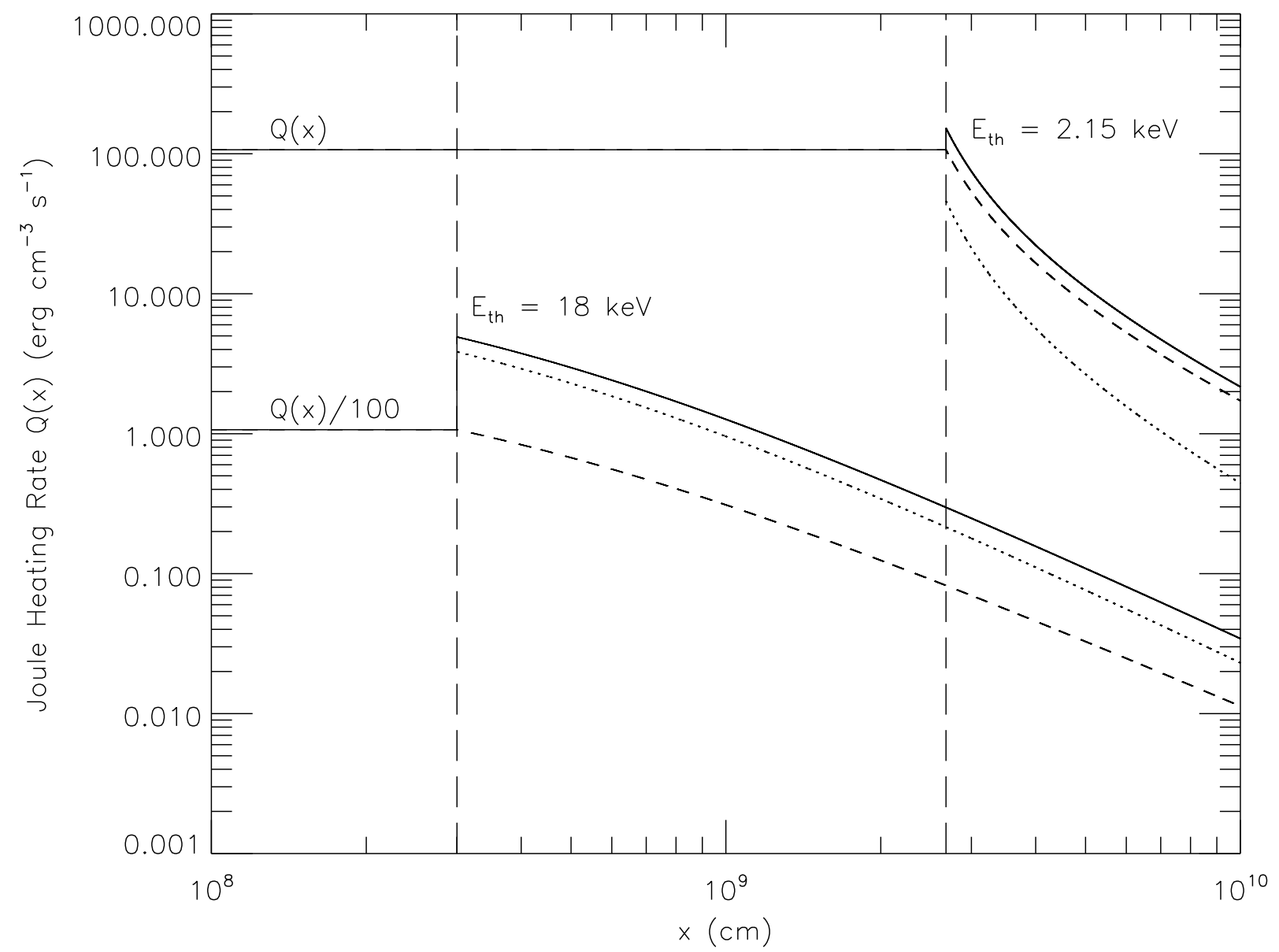

Fig. 3.- Volumetric heating rate, $Q(x)$, is plotted as a function of distance for $F_{e 0}=10^{19}$ electrons $\mathrm{s}^{-1} \mathrm{~cm}^{-2}, T=10 \mathrm{MK}, \lambda=20, \delta=5, E_{c}=20 \mathrm{keV}$, and two values of $E_{t h}$, $E_{t h}=2.5 k T=2.15 \mathrm{keV}$ and $E_{t h}=18 \mathrm{keV}$ (solid curves). The two vertical dashed lines show the value of $x_{r c}$ for the two values of $E_{t h}$. The dashed curves show the value of $Q(x)$ for Joule heating alone. The dotted curves show the heating due to thermalization of the electrons at $E_{t h}$ alone. The results for $E_{t h}=18 \mathrm{keV}$ are reduced by a factor of 100 to avoid overlapping curves. 


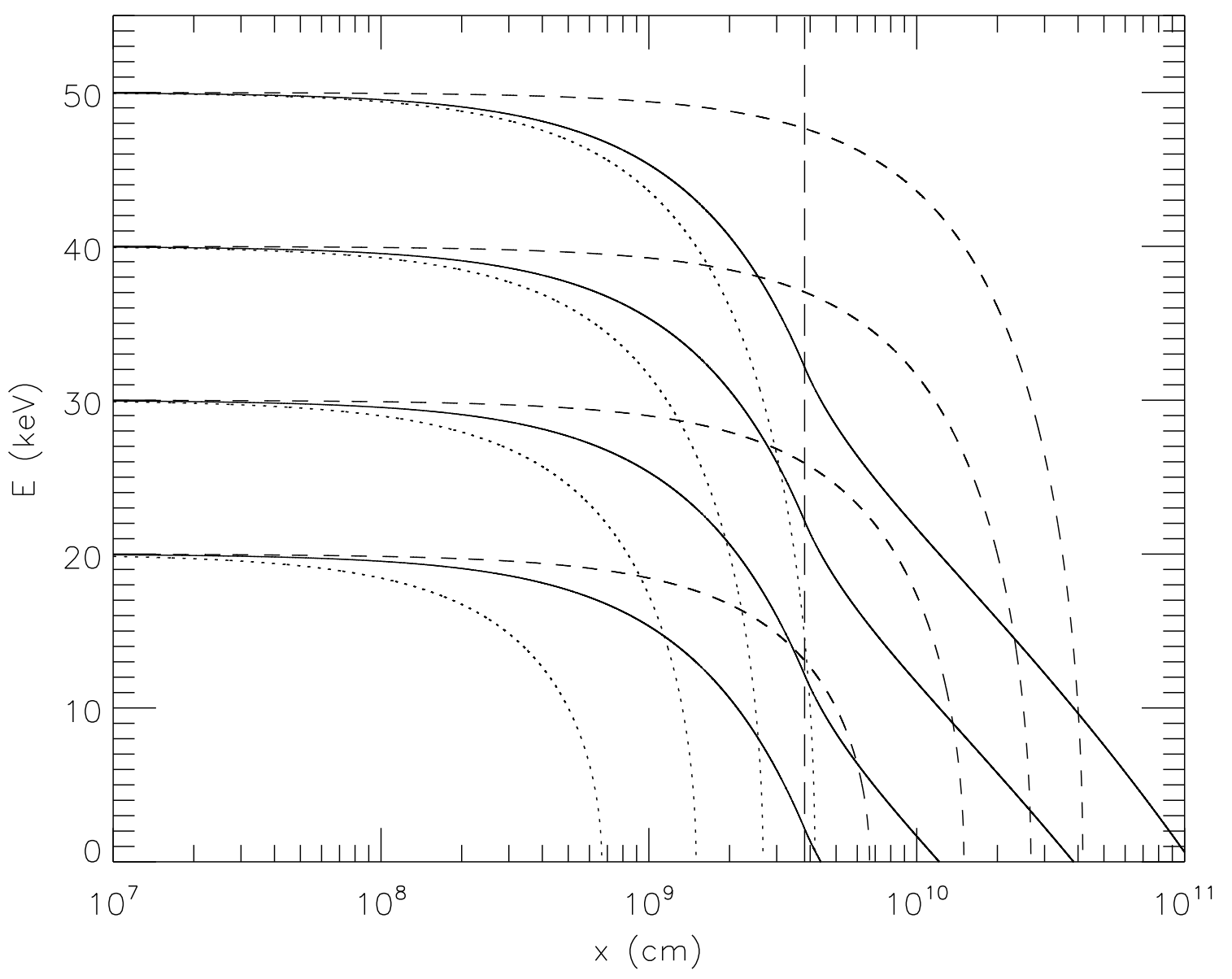

Fig. 4.- Comparison of return-current and collisional energy losses as a function of distance for electrons with four initial energies, $E_{0}=20,30,40$ and $50 \mathrm{keV}$. The solid curves show the decrease in electron energy resulting from return-current losses for $F_{e 0}=7 \times 10^{18}$ electrons $\mathrm{s}^{-1} \mathrm{~cm}^{-2}\left(P_{e 0}=3 \times 10^{11} \mathrm{erg} \mathrm{s}^{-1} \mathrm{~cm}^{-2}\right), T=10 \mathrm{MK}, \lambda=20, \delta=5, E_{c}=20 \mathrm{keV}$, and $E_{t h}=2.5 k T=2.15 \mathrm{keV}$. Collisional losses for $n=10^{10} \mathrm{~cm}^{-3}$ (dashed curves) and $n=10^{11} \mathrm{~cm}^{-3}$ (dotted curves) are shown. The vertical dashed line shows the value of $x_{r c}$. 


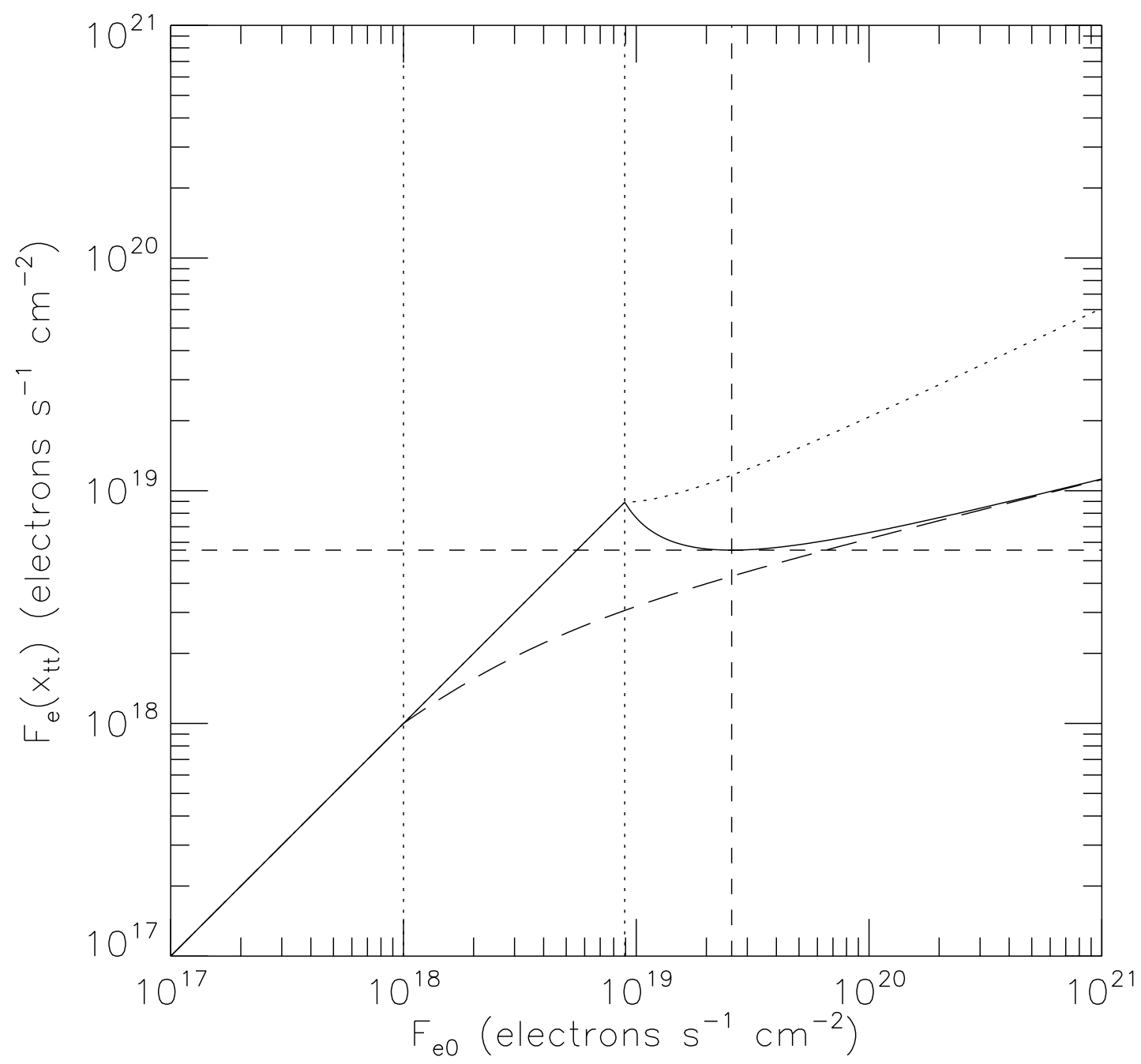

Fig. 5.- Electron flux density at the location of the thick-target footpoint, $x_{t t}$, is plotted as a function of the injected electron flux density for $x_{t t}=30 \mathrm{Mm}, T=10 \mathrm{MK}, \lambda=20, \delta=4$, $E_{c}=20 \mathrm{keV}$, and two values of $E_{t h}, E_{t h}=2.5 k T=2.15 \mathrm{keV}$ (solid curve) and $E_{t h}=18 \mathrm{keV}$ (long dashes). The two vertical dotted lines show the value of $F_{e 0}^{t t}$ for the two values of $E_{t h}$. The crossed horizontal and vertical lines (short dashes) show the value of $F_{e}^{\text {min }}\left(x_{t t}\right)$ and the value of $F_{e 0}$ at which this minimum occurs, respectively, for $E_{t h}=2.15 \mathrm{keV}$. The dotted curve is for $\delta=2.1$ and $E_{t h}=2.15 \mathrm{keV}$. 




Fig. 6. - X-ray brightness integrated over all photon energies above $20 \mathrm{keV}$ as a function of the injected electron flux density when energy losses in the thick-target region are dominated by collisional losses (Equation 37). Results are shown for five values of the injected electron density distribution power-law index, $\delta$. Other assumed parameter values are $x_{t t}=10 \mathrm{Mm}$, $T=10 \mathrm{MK}, \lambda=20, E_{c}=20 \mathrm{keV}$, and $E_{t h}=2.5 \mathrm{kT}=2.15 \mathrm{keV}$. The dotted vertical line shows the value of $F_{e 0}^{t t}$. 


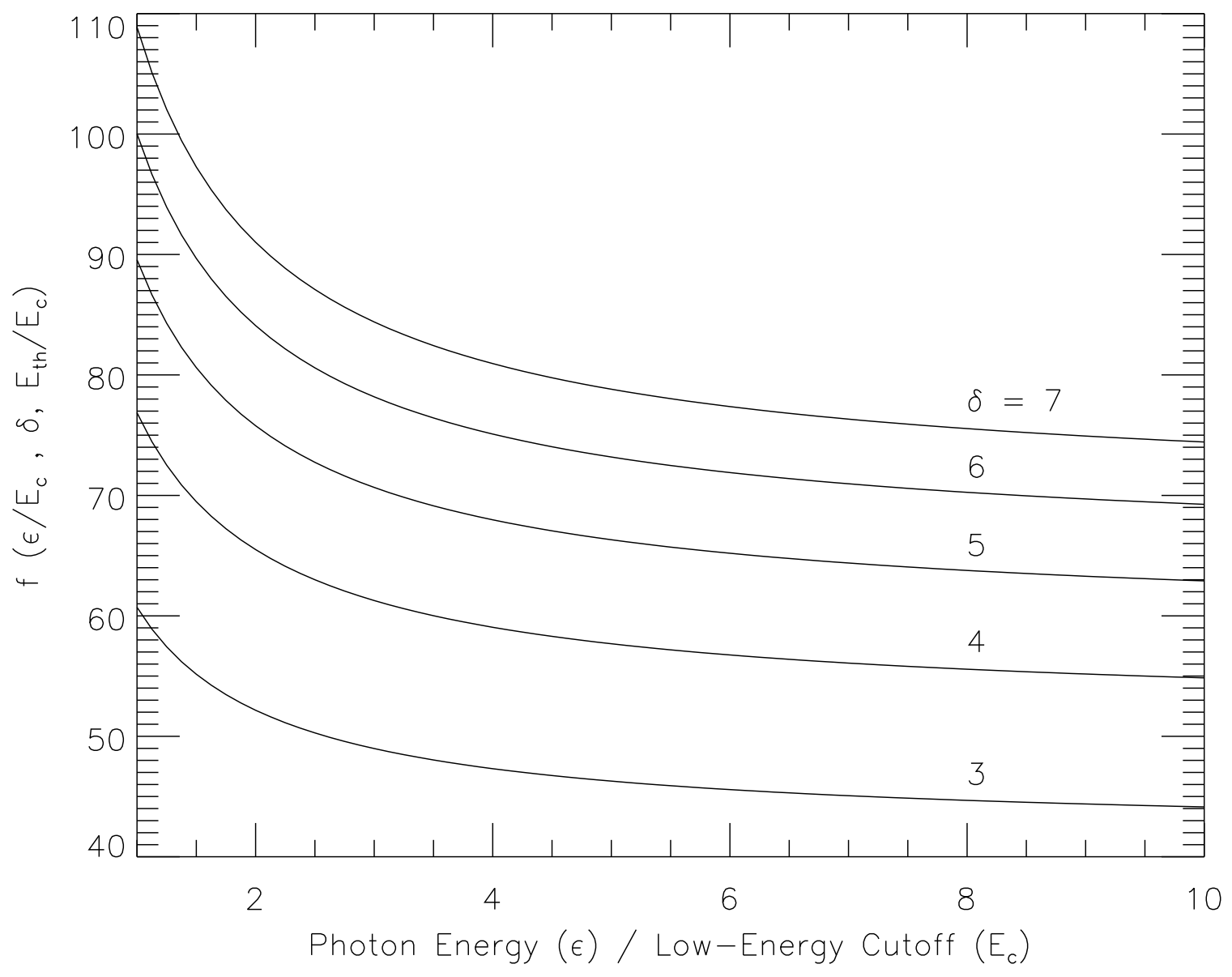

Fig. 7.- Integrated X-ray brightness function $f\left(\hat{\epsilon}, \delta, \hat{E}_{t h}\right)$ (Equation 41) verses $\hat{\epsilon}$ for five values of the electron power-law index $\delta$ and $\hat{E}_{t h}=0.108$. 


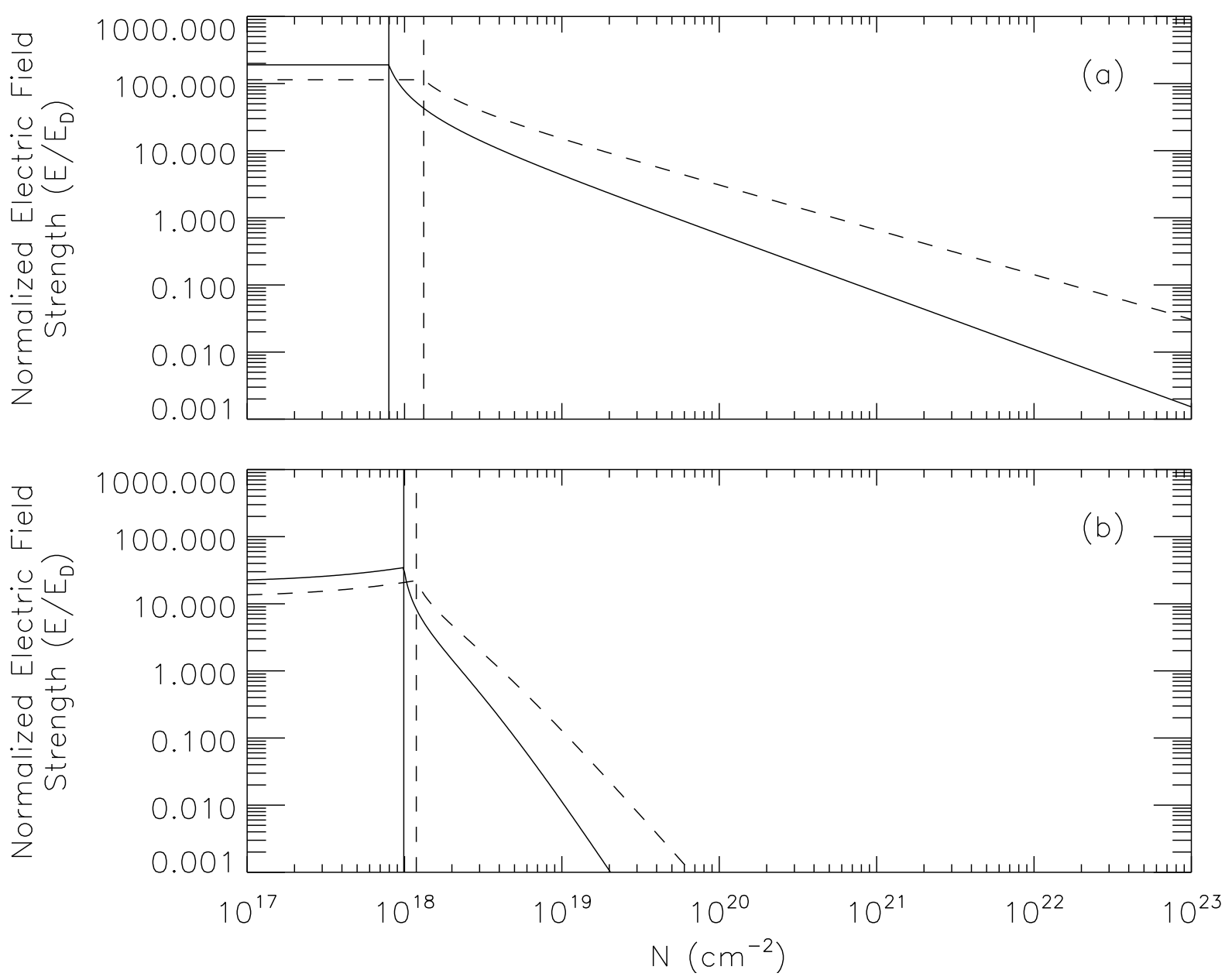

Fig. 8.- (a) Return-current electric field normalized to the Dreicer electric field vs. column density for $\delta=3$ (dashed curve) and $\delta=7$ (solid curve). The plasma temperature and density, derived for comparison with Figure 1(c) of Zharkova \& Gordovskyy (2006), are taken to have the constant values (independent of column density) $T=570 \mathrm{MK}$ and $n=$ $1 \times 10^{7} \mathrm{~cm}^{-3}$. The low-energy cutoff is $E_{c}=8 \mathrm{keV}, E_{t h}=0$, and the electron energy flux density is $1 \times 10^{12} \mathrm{erg} \mathrm{s}^{-1} \mathrm{~cm}^{-2}$. The vertical lines are the corresponding values of $N_{r c}=n x_{r c}$. (b) Normalized return-current electric field when the plasma density increases exponentially and the temperature decreases exponentially with distance (see Section 3.1). 


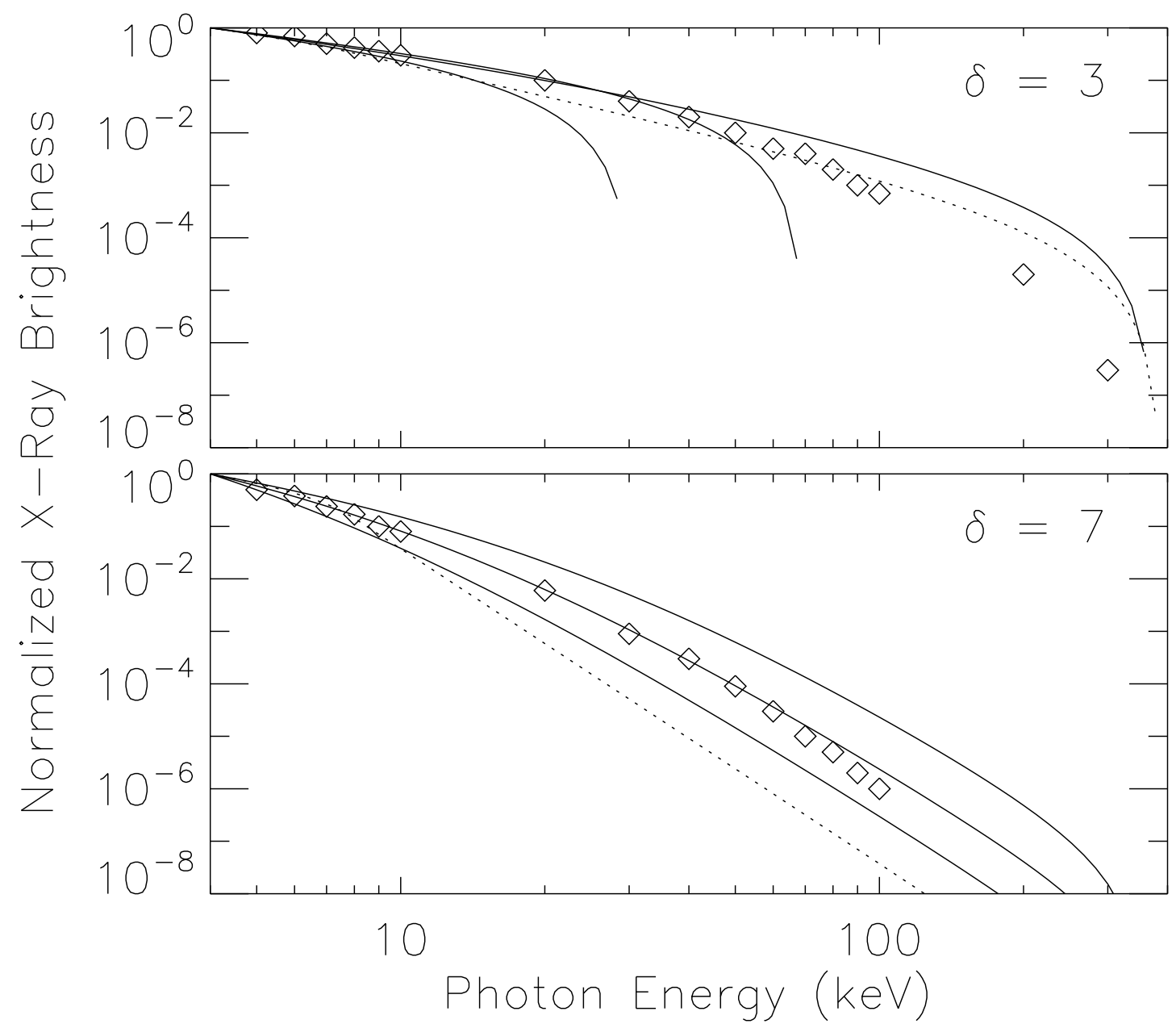

Fig. 9.- Thick-target X-ray brightness spectra, normalized to their value at $4 \mathrm{keV}$, computed using Equation 42 for $\delta=3$ (top panel) and $\delta=7$ (bottom panel) and several values of $V_{t t}$. For comparison with spectra from Figure 10(b) of Zharkova \& Gordovskyy (2006), $E_{c}=8 \mathrm{keV}$ and $E_{u p p}=384 \mathrm{keV}$. The symbols (diamonds) are points from the spectra in Figure 10(b). The dotted curves are for $V_{t t}=0 \mathrm{kV}$. The solid curves in the upper panel $(\delta=3)$ are, from top to bottom, for $V_{t t}=15,315$, and $354 \mathrm{kV}$. The solid curves in the lower panel $(\delta=7)$ are, from top to bottom, for $V_{t t}=6,12$, and $24 \mathrm{kV}$. 


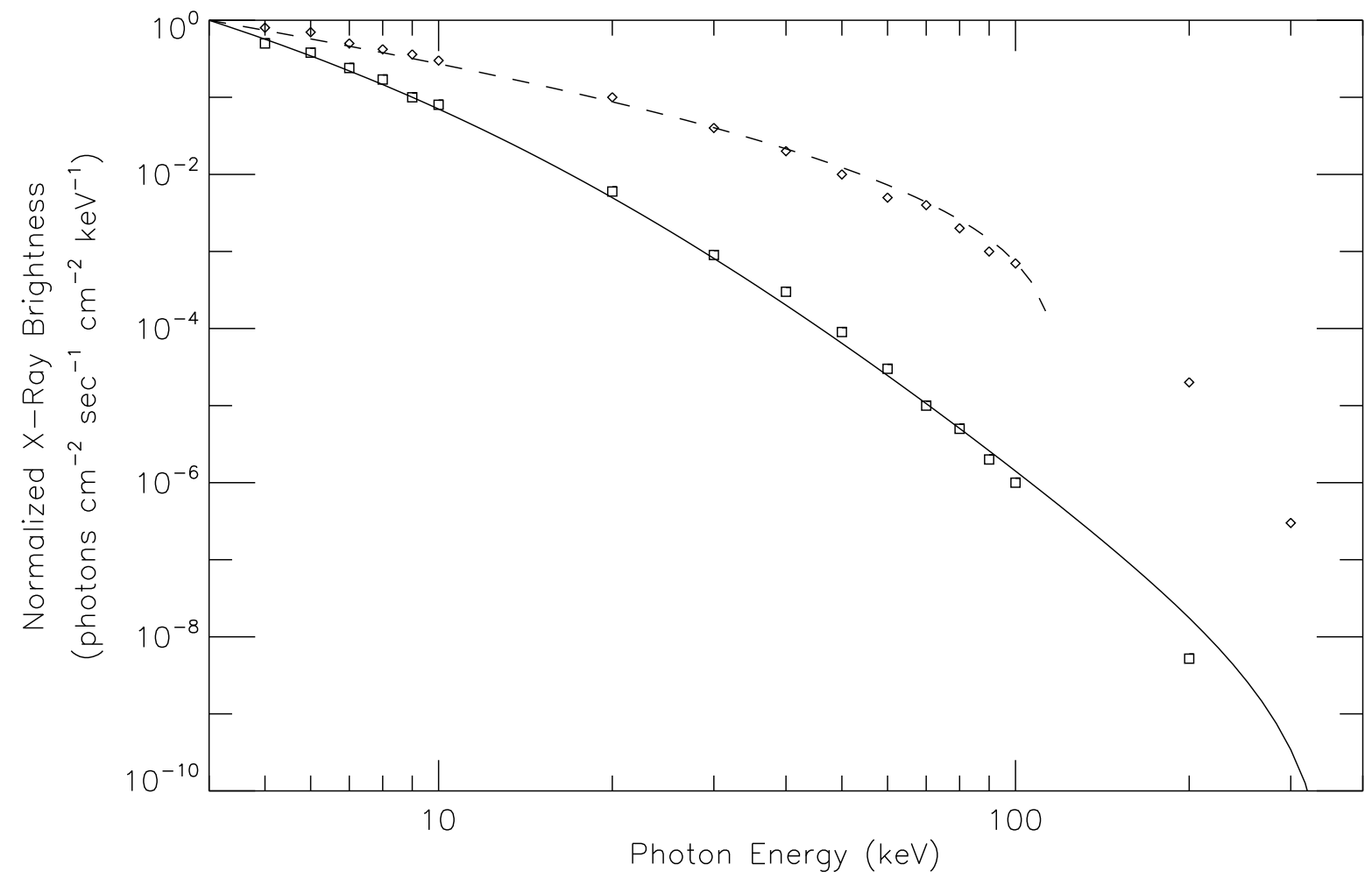

Fig. 10.- Thick-target X-ray brightness spectra, normalized to their value at $4 \mathrm{keV}$, computed for $E_{c}=8 \mathrm{keV}$ and $E_{u p p}=384 \mathrm{keV}$ using the Haug (1997) bremsstrahlung cross section, for comparison with spectra from Figure 10(b) of ZG06. The symbols are from Figure 10(b) and the curves are computed using the 1-D distribution function with returncurrent losses (Equation 12). The diamond symbols and dashed curve are for $\delta=3$. The square symbols and solid curve are for $\delta=7$. These fits give best values for the potential drop at the top of the thick-target region of $V_{t t}=130 \mathrm{kV}$ for $\delta=3$ and $V_{t t}=14 \mathrm{kV}$ for $\delta=7$. 


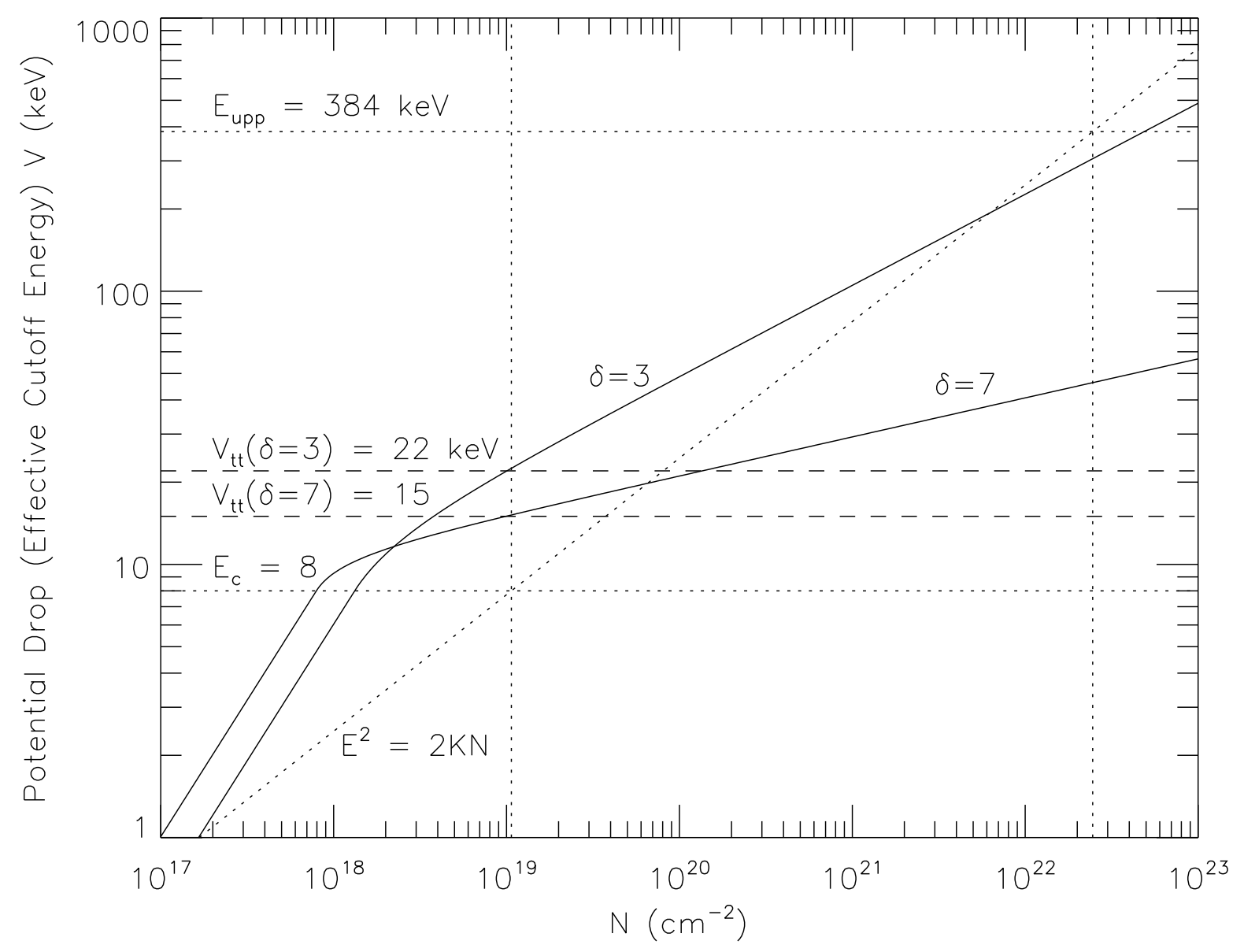

Fig. 11.- The computed potential drop $V$ as a function of column density $N$ for $\delta=3$ and $\delta=7$ (solid curves) for comparison with the spectral fit results in Figure 10. The plasma density and temperature are assumed to be everywhere $n=1 \times 10^{7} \mathrm{~cm}^{-3}$ and $T=570 \mathrm{MK}$, the values deduced from Figure 1(c) of ZG06. $E_{c}=8 \mathrm{keV}$ and $E_{\text {upp }}=384 \mathrm{keV}$ (dotted horizontal lines) are the lower and upper cutoffs to the electron distribution. The thicktarget region is between the two dotted vertical lines. $V_{t t}$ is the value of the potential drop at the top of the thick-target region (dashed horizontal lines). The dotted diagonal line shows the values of $N$ at which the energy of electrons of energy $V$ is reduced to zero by collisional losses. 


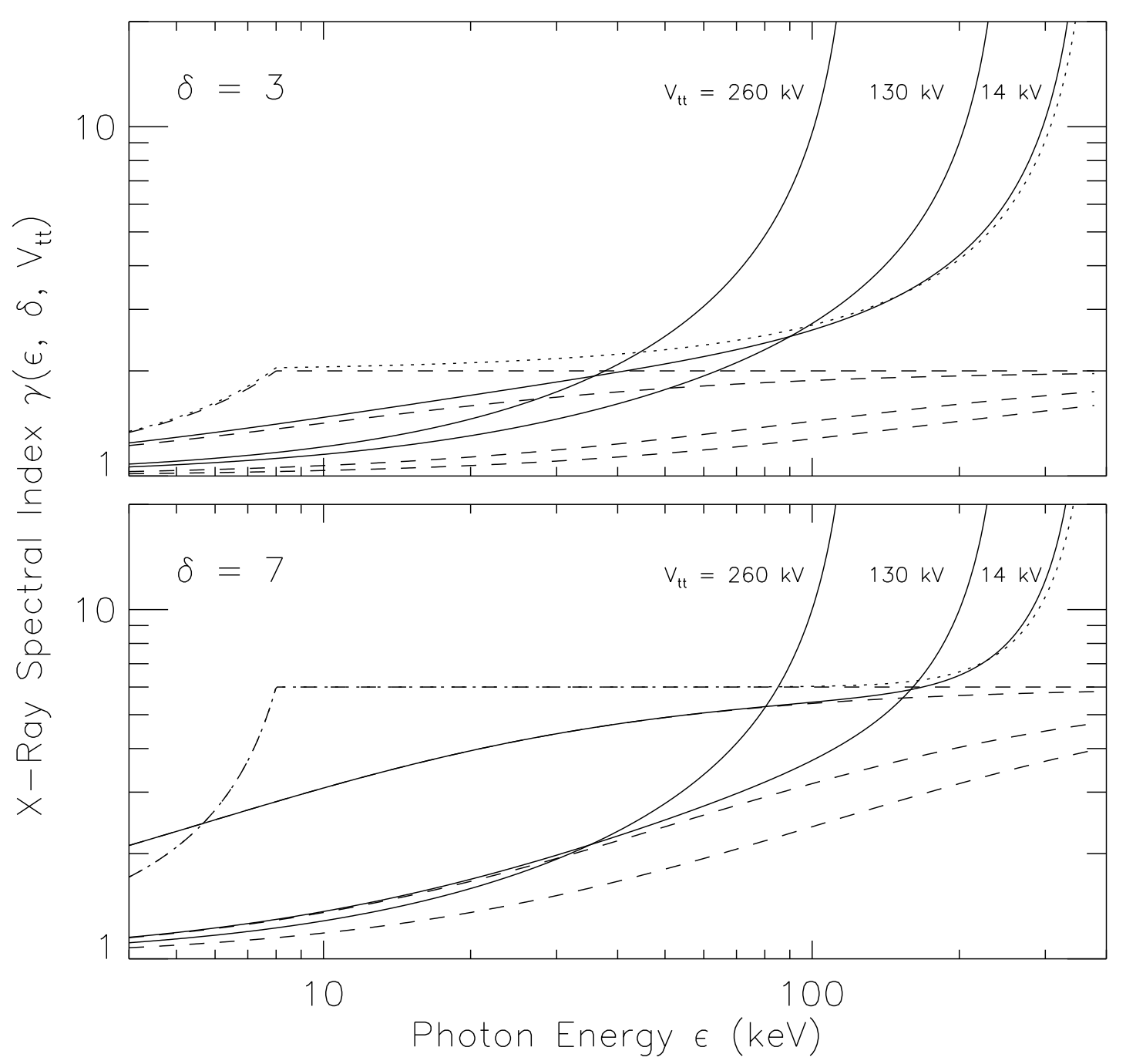

Fig. 12.- Thick-target X-ray spectral index plotted as a function of photon energy for an injected electron flux density distribution with a power-law index of 3 (top panel) and 7 (bottom panel), computed from Equations 43 and 44 with $E_{c}=8 \mathrm{keV}$. The dotted and solid curves are for $E_{\text {upp }}=384 \mathrm{keV}$. The dashed curves are for no high-energy cutoff $\left(E_{\text {upp }} \rightarrow \infty\right)$. The dotted and corresponding dashed curves are for $V_{t t}=0 \mathrm{kV}$. The solid curves and their corresponding dashed curves are for $V_{t t}=14,130$, and $260 \mathrm{kV}$. 

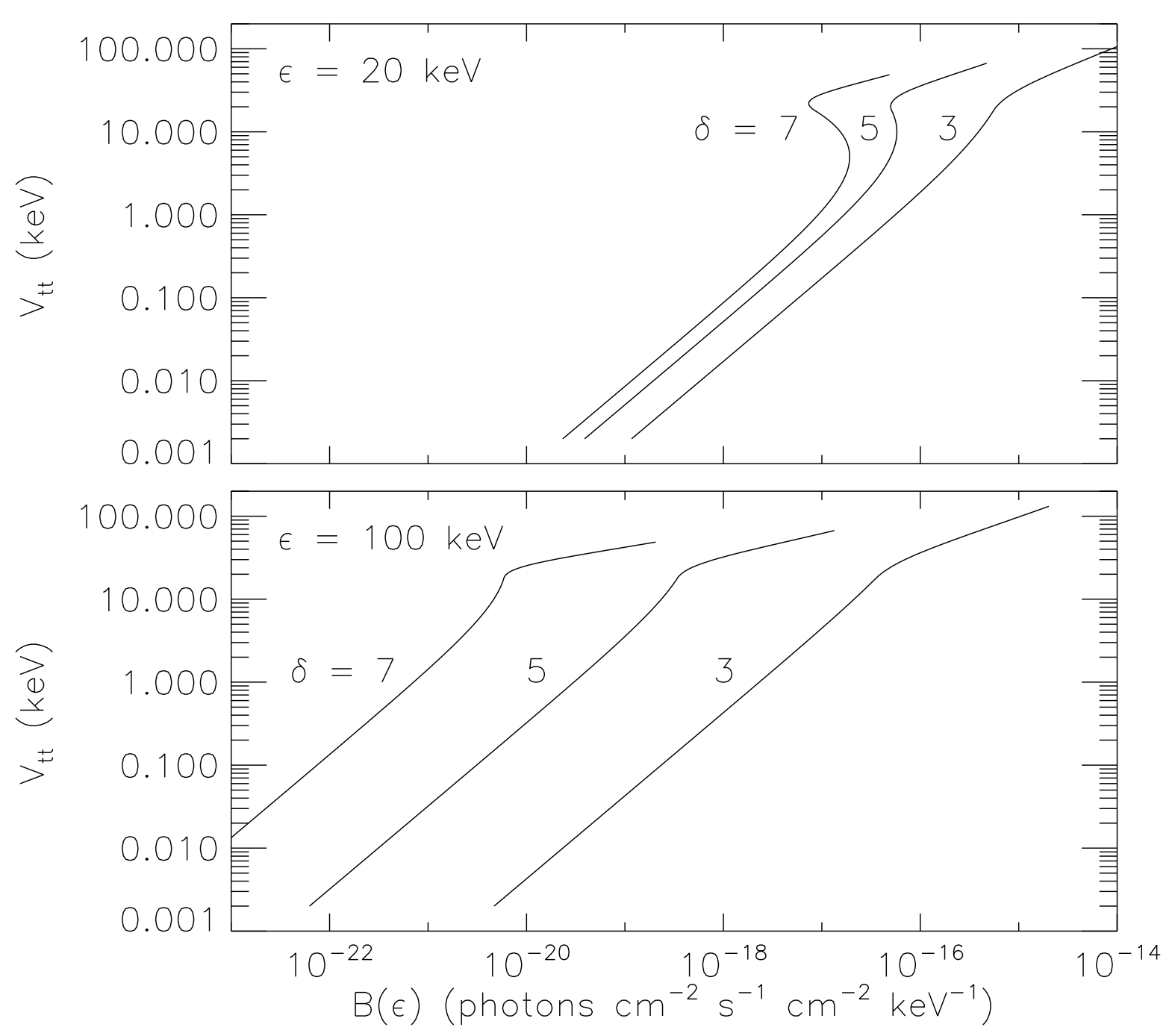

Fig. 13. - Variation of the potential drop at the top of the thick-target region, $V_{t t}$, with the thick-target X-ray brightness, $B(\epsilon)$, as the injected electron flux density increases from $10^{15}$ to $10^{21}$ electrons $\mathrm{s}^{-1} \mathrm{~cm}^{-2}$. The top panel shows the X-ray brightness at $20 \mathrm{keV}$ and the bottom panel shows the X-ray brightness at $100 \mathrm{keV}$. Each panel shows curves for three values of the power-law index of the injected electron flux density distribution: $\delta=3,5$, and 7. The curves are computed using Equations 37 and 16 with $T=10 \mathrm{MK}, E_{c}=20 \mathrm{keV}$, $E_{t h}=2.15 \mathrm{keV}, x_{t t}=3 \times 10^{9} \mathrm{~cm}$, and no high-energy cutoff. 

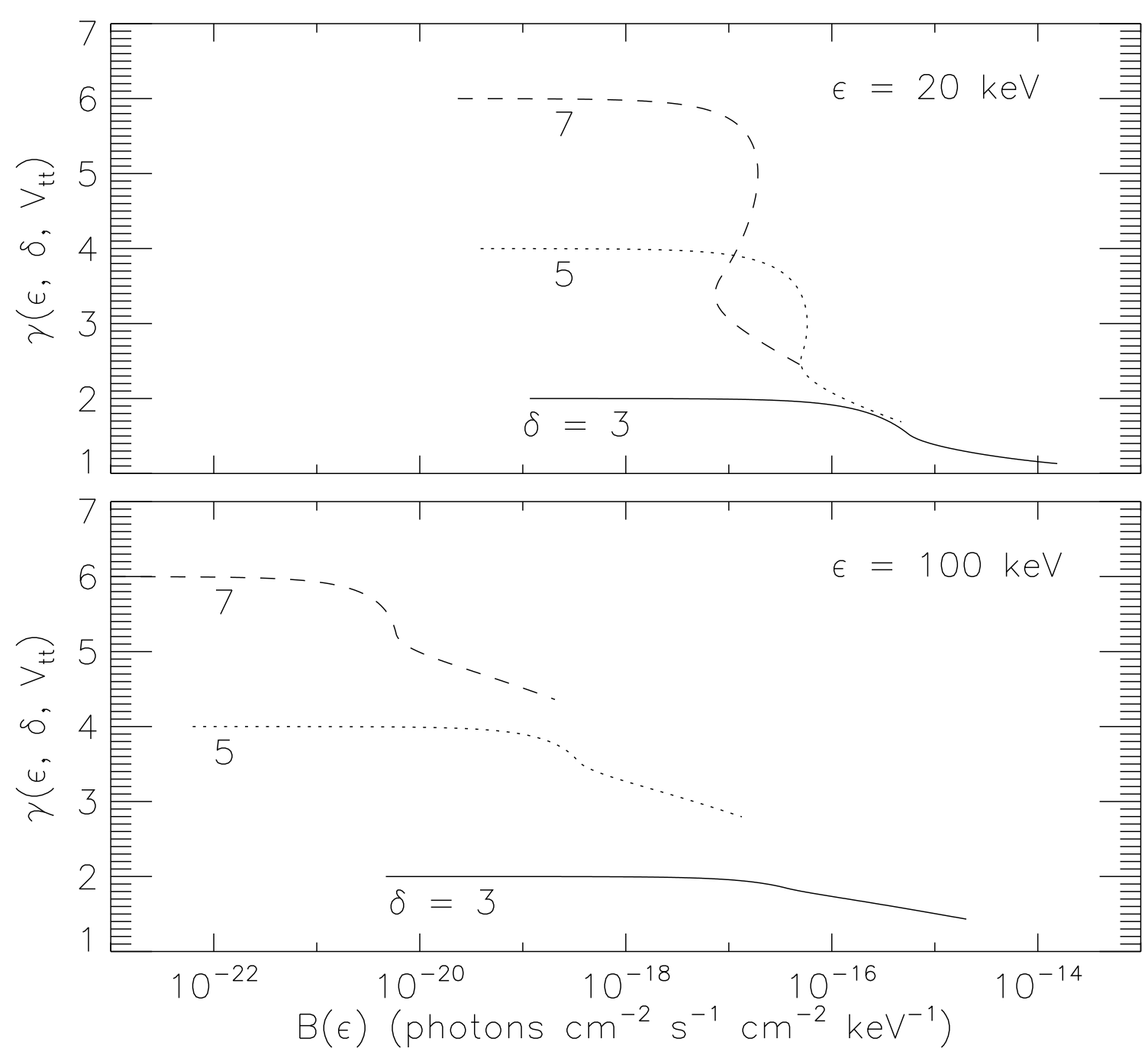

Fig. 14. - Variation of the thick-target spectral index, $\gamma(\epsilon)$, with the thick-target X-ray brightness, $B(\epsilon)$, as the injected electron flux density increases from $10^{15}$ to $10^{21}$ electrons $\mathrm{s}^{-1} \mathrm{~cm}^{-2}$. The top panel shows the X-ray brightness and spectral index at $20 \mathrm{keV}$, and the bottom panel shows the X-ray brightness and spectral index at $100 \mathrm{keV}$. Each panel shows curves for three values of the power-law index of the injected electron flux density distribution: $\delta=3,5$, and 7. The curves are computed using Equations 37 and 44 with $T=10 \mathrm{MK}, E_{c}=20 \mathrm{keV}, E_{t h}=2.15 \mathrm{keV}, x_{t t}=3 \times 10^{9} \mathrm{~cm}$, and no high-energy cutoff. 


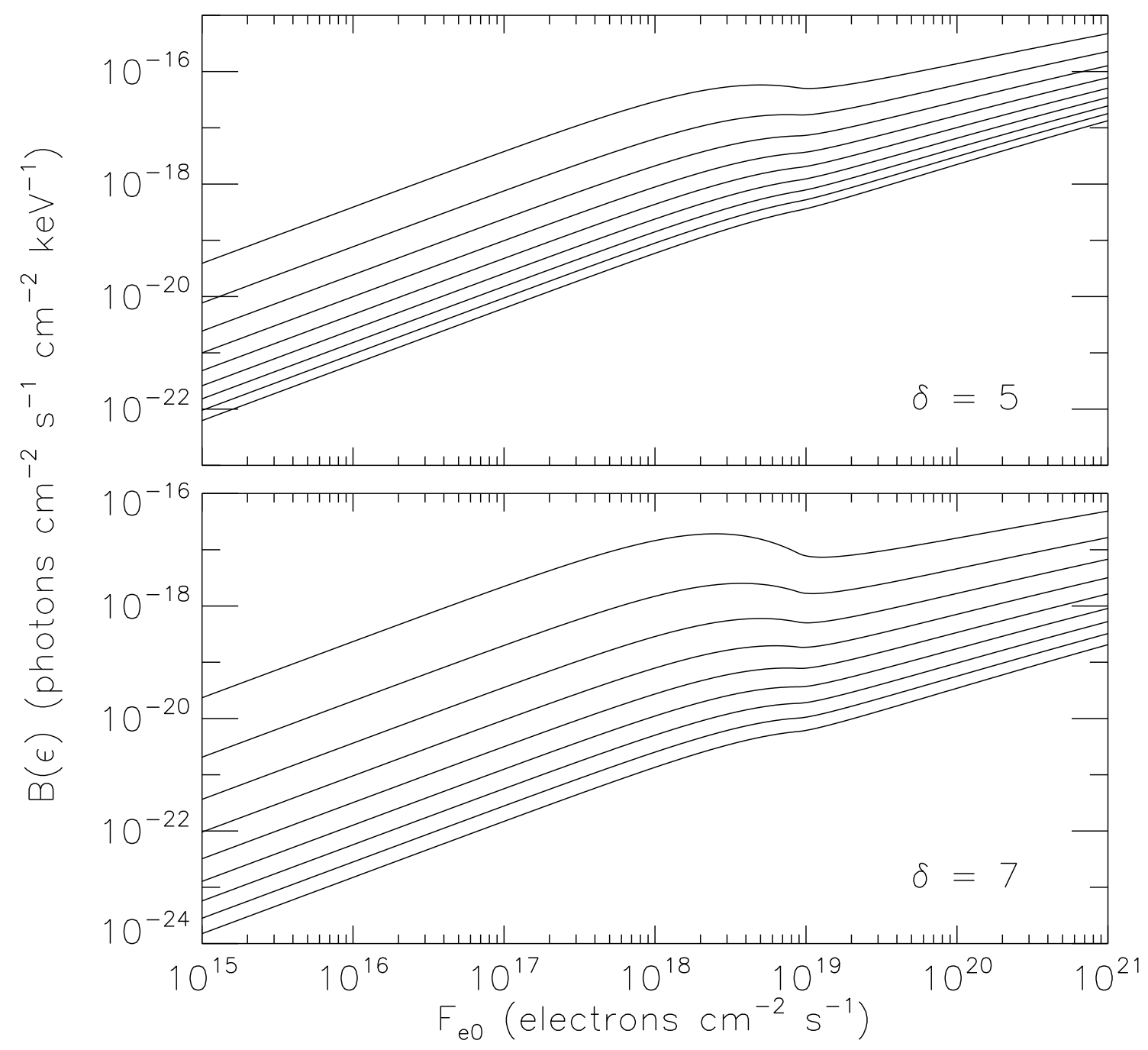

Fig. 15.- Variation of the thick-target photon brightness at energy $\epsilon, B(\epsilon)$, with the injected electron flux density, $F_{e 0}$. The top panel shows the X-ray brightness for $\delta=5$, and the bottom panel shows the X-ray brightness for $\delta=7$. Each panel shows curves for nine values of the photon energy, starting at $\epsilon=20 \mathrm{keV}$ (top curve) and increasing in steps of $10 \mathrm{keV}$ to $\epsilon=100 \mathrm{keV}$ (bottom curve). The curves are computed using Equations 37 and 16 with $T=10 \mathrm{MK}, E_{c}=20 \mathrm{keV}, E_{t h}=2.15 \mathrm{keV}, x_{t t}=3 \times 10^{9} \mathrm{~cm}$, and no high-energy cutoff. 